\title{
LES STRUCTURES DE VÉGÉTATION
}

Le présent mémoire est basé principalement sur une de mes contributions au Ier Séminaire International de Géographie, organisé par le Professeur Orlando Ribeiro et ses collaborateurs, à Lisbonne, du 12 au 17 mars 1967. Toutefois, il contient un certain nombre d'éléments nouveaux, entre autres une réforme de la nomenclature des types-de-formation ou. protoformations.

Il s'agira d'abord d'établir les critères analytiques qui servent à caractériser toute végétation et qui se rapportent à la composition, à la structure et au dynamisme. Les formes et les fonctions que révèlent ces analyses relèvent de déterminants divers, tels la phylogéne, le climat, le sol et l'histoire.

Les dimensions structurales de la végétation, à diverses échelles, peuvent se mesurer en combinant divers critères qui seront passés en revue. L'importance et l'utilité relatives de ces critères varieront selon les besoins de la classification et de la définition des unités structurales. Deux ordres fort distincts sont reconnus dans la présente contribution: les protoformations, qui sont purement structurales et aucunement écologiques ou géographiques, et les classes-de-formation, qui sont nettement bioclimatiques.

LES TROIS ÉLÉMENTS DE LA VÉGÉTATION

Une station concrète de végétation, par exemple une chênaie d'yeuse dans la Serra da Arrábida (Portugal), une forêt de lauriers à Madère ou une érablière sur l'Ile de Montréal (Canada), se prête à une analyse plus ou moins 
poussée, selon les besoins de l'investigateur. Pour bien des raisons et pour bien des fins, quelque chose de moins qu'une analyse complète sera satisfaisant. C'est pourtant en envisageant les exigences de cette connaissance détaillée qu'on pourra le mieux encadrer celles des investigateurs autres que l'écologiste.

Je commencerai donc par tracer les lignes d'un inventaire proprement écologique et phytosociologique afin de mieux situer les définitions, surtout structurales, qui importent davantage au géographe.

Le tableau I est un relevé phytosociologique fait dans la forêt de la Serra da Arrábida, selon la méthode de l'Ecole de Montpellier (voir Braun-Blanquet, 1928, 1932, 1964) quelque peu modifiée (DANSEREAU, 1957). C'est un échantillonnage limité à un quadrat de $10 \times 20 \mathrm{~m}$, dont les conditions topographiques et édaphiques sont brièvement notées.

Composition - On y a trouvé en tout 15 espèces de plantes. Cette simple énumération nous apprend déjà quelque chose, puisque certaines des qualités que possèdent les espèces leur sont propres dans toute leur aire: leur morphogenèse, leur physiologie, leur génétique et surtout des exigences particulières qui font de certaines d'entre elles de bons indicateurs. Ainsi le chêne-vert (Quercus ilex) révèle-t-il la présence de calcaire, le laurier-tin (Viburnum tinus) celle d'un sol frais et relativement profond; le Sedum le passage récent de l'homme; le Rubus ulmifolius la pénétration de la lumière jusqu'au niveau du sol. D'autre part le plus grand nombre de ces espèces est nettement sciophile et mésophile, c'est à-dire capable de se perpétuer dans l'ombre et sur un site où la provision d'eau est relativement plus constante qu'ailleurs dans le paysage.

D'autres qualités encore s'attachent à chaque espèce indépendamment du rôle que jouent ici les populations locales et en quelque sorte préalablement à leur établissement. Une panoplie de ces qualifications apparaît au tableau II: la forme biologique, les caractères de la feuille, le moyen apparent de dispersion, etc. Des comparaisons que j'ai faites ailleurs (1961, 1968) entre diverses associations végétales montrent des différences souvent considérables quant à chacun de ces

\section{TABLEAU I}

Relevé sur le terrain dans une forêt d'yeuse (chêne-vert), le 12 mars 1967, à la Serra da Arrábida, aux abords du Caminho da Mata Coberta. Forêt très ancienne, traversée par un chemin de pèlerins; peut-être jamais coupée. Propriété du Duc de Palmela. Pente, envircn 25", exposition est; superficie du quadrat: $200 \mathrm{~m}^{2}$. Sol rocailleux limoneux, compact; humus à la surface. Les indices en face des noms d'espèces donnent la classe de couverture selon l'échelle de DANSERFAU (1957, pp. 192-194): $1.1=$ moins de $20 \%$ de couverture totale, également repartie; 1.2: même couverture distribuée inégalement: $\mathrm{L}=$ liane; $+=1-4 \% ; 3=41-60 \% ; .<1 \%$.

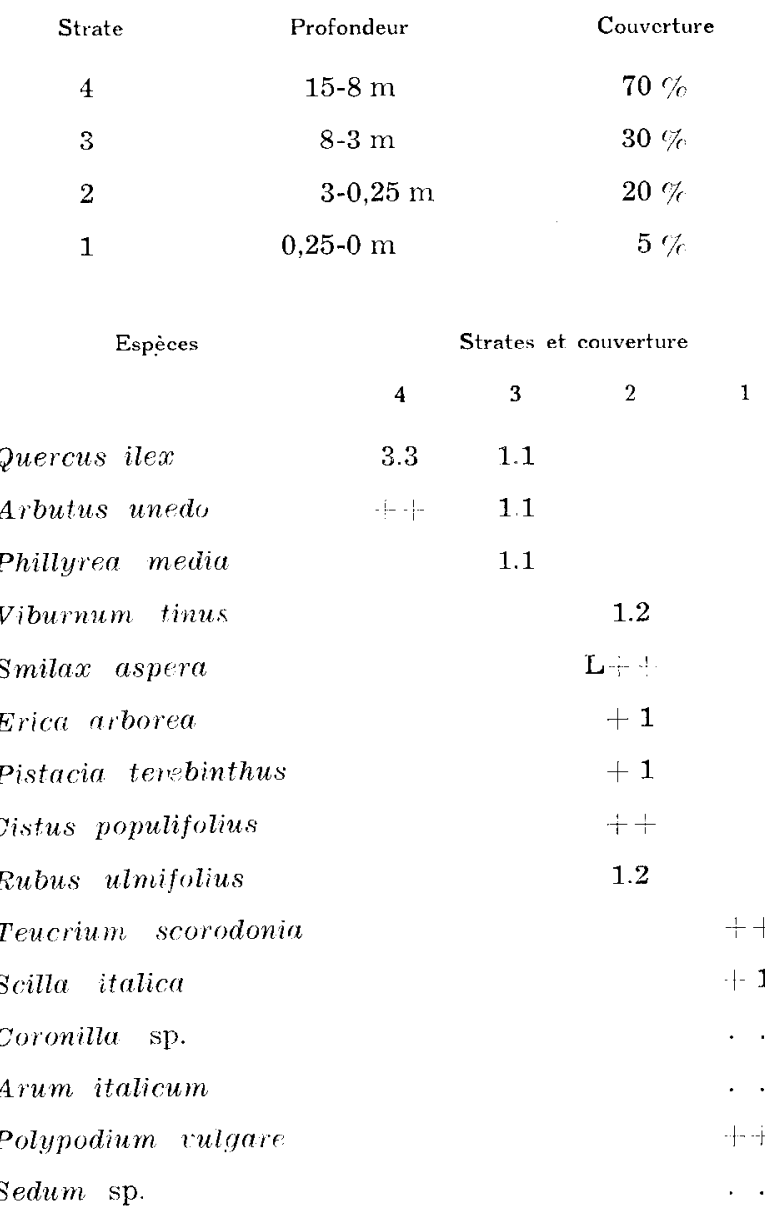


douze critères. Ainsi la forêt d'yeuse a le même pourcentage d'hémicryptophytes qu'une forêt de podocarpes de la NouvelleZélande et en a deux fois moins qu'une aulnaie canadienne et une savane portoricaine. Elle a $60 \%$ d'espèces sempervirentes; il n'y en a que $10 \%$ dans l'aulnaie, mais $100 \%$ dans la forêt néozélandaise et $36 \%$ dans la savane. Elle comporte un peu plus de $30 \%$ d'espèces mésophiles, à peu près la même proportion que l'aulnaie, mais la forêt de podocarpes en compte $\mathbf{7 6 \%}$ et la savane aucune! Quant aux moyens apparents de dispersion, la chênaie portugaise compte $35 \%$ de fruits charnus, la même chose que l'aulnaie; la forêt de podocarpes en compte $45 \%$ et la savane seulement $14 \%$. En revanche, la chênaie ne compte aucune espèce à appendice ailé ou plumeux; la forêt néozélandaise en compte très peu $(3,5 \%)$, l'aulnaie, davantage $(15 \%)$ et la savane portoricaine, énormément plus $(22 \%)$.

Or, cette liste qualitative nous renseigne sur la flore des $200 \mathrm{~m}^{2}$ examinés, mais elle ne constitue nullement une description de la végétation aussi longtemps que les populations des espèces présentes n'ont pas été groupées selon leur importance relative ou même leur importance absolue. Il ne s'agit pas, dans le présent contexte, de comparer les méthodes d'inventaire, qui sont très diverses et que chaque école défend en vertu des questions particulières qu'elle se pose. Il y aurait moyen de procéder, par exemple, par ablation totale de toute la matière végétale que contient la place-échantillon (ou le quadrat) et de peser la biomasse entière, ou même chaque population spécifique. Ou encore on pourrait déterminer l'abondance en comptant les individus (ou les tiges) de chaque population spécifique; ou bien subdiviser l'aire totale en carrés plus petits afin de déterminer le nombre de carrés où chacune est présente, soit la fréquence. Ou bien, comme il a été fait au tableau I, on adoptera une échelle basée d'abord sur la projection verticale (ou couverture) pour les unités à fort recouvrement et sur l'abondance pour les espèces plus clairsemées: cet «estimé global», recommandé par BRAUNBLANQUET $(1928,1932,1964)$ a été très généralement utilisé en Europe continentale.

Pour ma part je l'ai modifié quelque peu (1957, tableaux 3-13 et $3-14$ et figures $3-39$ et $3-40$ ), mais très géné- reusement appliqué depuis l'Arctique jusqu'aux Tropiques. Le tableau I fait done voir la couverture de chacune des 15 espèces d'après un estimé visuel.

Structure - Or, cette indication quantitative est répétée dans chacune des strates où chaque espèce se trouve présente. Ainsi le Quercus ilex et l'Arbutus unedo occupent des places fort différentes dans la $4^{\prime \prime}$ et la $3^{\circ}$ strates. Il se trouve que chacune des autres espèces est confinée à une seule strate. Ceci nous introduit à la notion de structure. En effet il n'aura pas suffi de noter la présence du Quercus ilex, dont on peut fort bien savoir qu'il est une mésophanérophyte, c'est-à-dire un arbre de taille moyenne, car la population locale pourrait aussi bien consister en plantules de 25 centimètres de hauteur, et la végétation qui résulterait (même si la plante était abondante ou avait une forte couverture) ne mériterait guère de s'appeler une forêt.

Chacun croit savoir ce qu'est une forêt et ce qu'est un arbre. Pourtant, l'examen des définitions des botanistes, des géographes, des forestiers eux-mêmes ne nous satisfont guère plus que celle des lexicographes. Et c'est là une des raisons qui nous font poser à neuf la question: quels sont les éléments de la structure de la végétation? C'est demander une définition plus rigoureuse de vieilles expressions telles que prairie, pelouse, fourré, steppe, etc. Ou encore c'est réclamer une nouvelle classification, libre des connotations climatiques de «savane», «steppe», ou géographiques de «toundra», «désert», etc. Par conséquent, au lieu de partir de cette nomenclature empirique, il vaudra mieux rechercher les pièces fondamentales de la structure de toute végétation et se demander ensuite comment ces pièces sont susceptibles de s'assembler.

Dans plusieurs contributions précédentes (surtout 1951, 1958, 1968), j'ai discuté ces éléments, proposé une méthode graphique pour leur assemblage et finalement une classification des principales structures que j'ai appelées «typesde-formation». Je reprends ici cette méthode: le tableau III et les figures 1 à 4 les font voir.

Il convient peut-être d'offrir ici une brève démonstration de cette méthode. Le tableau II contient toutes les données 

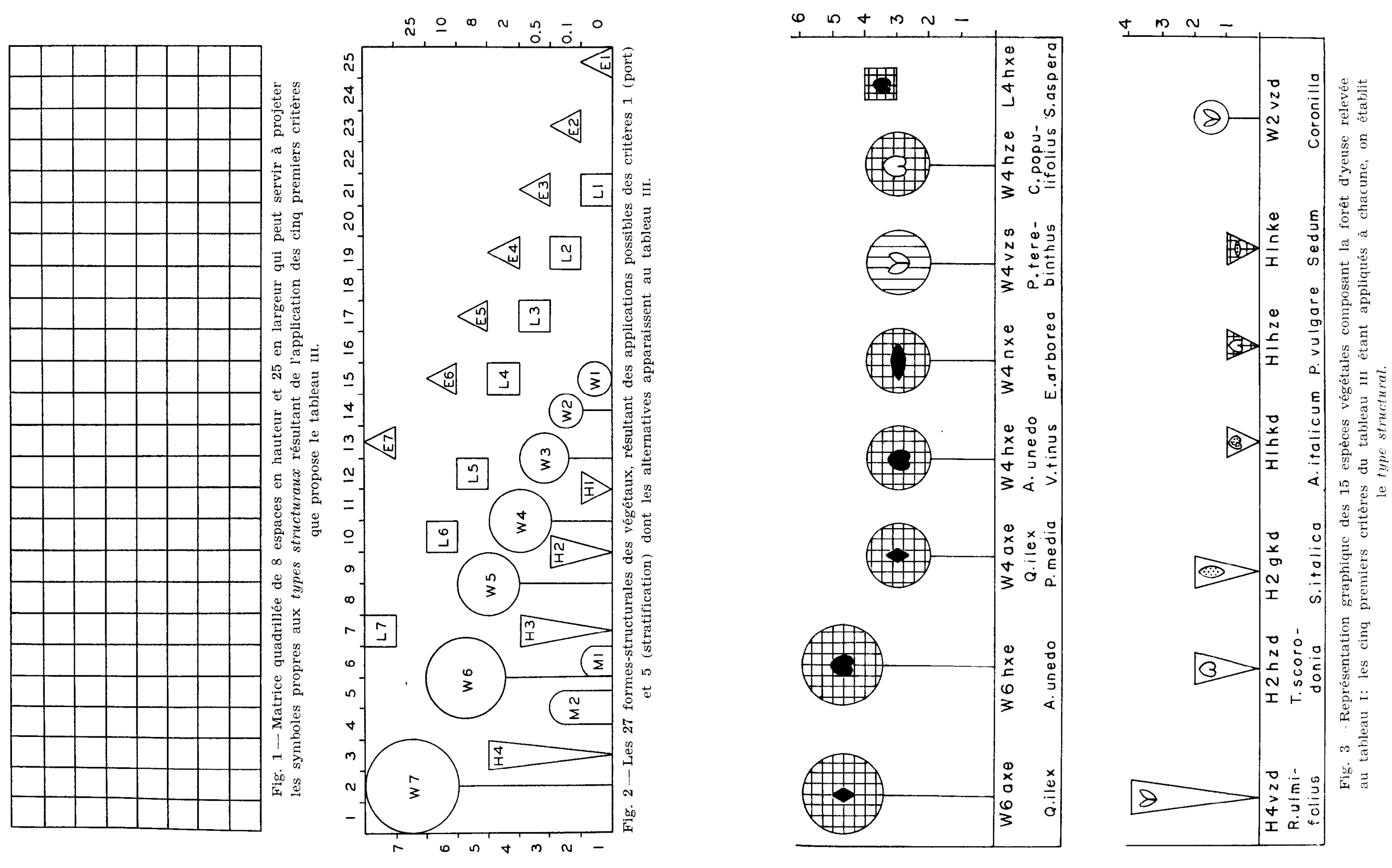


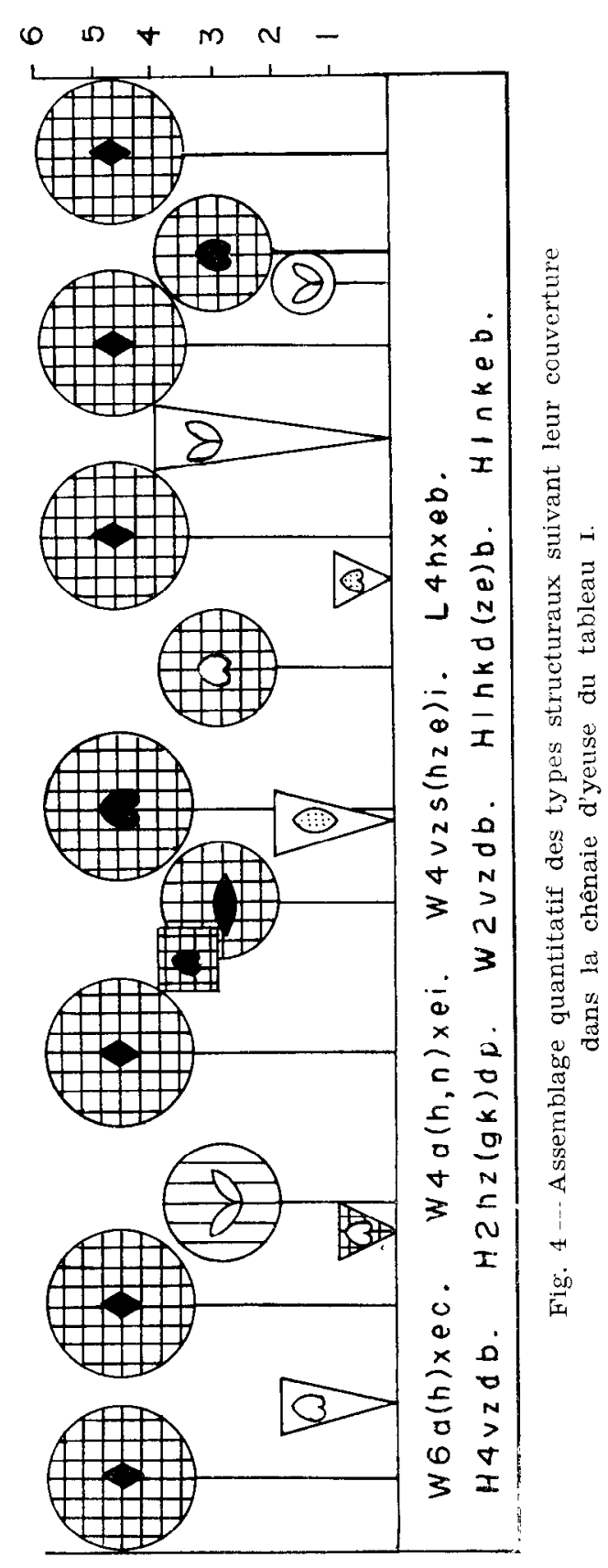


Analyse des caractéristıques de 15 espèces présentes dans le quadrat relevé au tableau I. Les symboles s'expliquent comme suit. 1. La forme biologique est selon RaUnkiaer (1934) et DANSEREAU (1959): Pm: mésophanérophyte; Pp: microphanérophyte; Pn: nanophanérophyte; Ps: phanérophyte grimpante; $\mathrm{Ch}$ : chamæphyte; Hs: hémicryptophyte rameuse; Hr: hémicryptophyte à rosette; G: géophyte. 2, 4, 5: voir tableau III 2. Port et stratification, voir tableau III, 1 et 5. 3. Grandeur de feuille en $\mathrm{mm}^{2}$ selon RAUnkiAER (1934): 1 (moins de 25 mm²), 2 (25-225), 3 (225-2025), 4 (2025-18 225), 5 (18 225-164 025), 6 (plus de 164025). 4. Type et texture de feuille, voir tableau III, 2 et 3.5 . Saisonnalité, 6. Rythme: Y: année entière; V: vernal; $\mathrm{E}$ : estival; $\mathrm{S}$ : sérotinal; A: automnal; $\mathrm{H}$ : hiémal. (La majuøcule se rapporte à l'activité végétative, la minuscule à l'activité sexuelle). 7. Type biocénotique: R: plantes enracinées; E: épiphytes. 8. Valence écologique: X: xérophile; M: mésophile H: hygrophile; $\mathrm{s}=$ sub-. 9. Type métabolique: A: annuelles; B: vivaces à cycle court; B': vivaces à croissance lente; C: vivaces à réserves annuelles; D: vivace traçante; $\mathrm{E}$ : plantes accumulatrices de réserves; F: plantes à forte capacité de réserve; G: multicentenaire. 10. Réinvestissement: f: fréquence; a: abondance; e: assimilabilité. (Les majuscules indiquent de fortes valeurs, les minuscules de faibles valeurs). 11. Types de diaspores (d'après DANSEREAU et LEMS, 1957): 1: auxochore; 2: cyclochore; 3: ptérochore; 4: pogonochore; 5: desmochore; 6: sarcochore; 7: sporochore; 8: sclérochore; 9: barochore; 10: ballochore. 12. Elément floristique, basé sur la distribution totale de l'espèce.

\begin{tabular}{|c|c|c|c|c|c|c|c|c|c|c|c|c|c|}
\hline Espèces & & $\begin{array}{c}1 \\
\begin{array}{c}\text { Forme } \\
\text { biologique }\end{array}\end{array}$ & $\begin{array}{c}2 \\
\begin{array}{c}\text { Port } \\
\text { stratifi- } \\
\text { cation }\end{array}\end{array}$ & $\begin{array}{c}3 \\
\text { Grandeur } \\
\text { de } \\
\text { feuille }\end{array}$ & \begin{tabular}{c}
\multicolumn{1}{c}{4} \\
\multicolumn{1}{c}{ Type } \\
\& texture \\
de feuille
\end{tabular} & $\begin{array}{c}5 \\
\text { Saison- } \\
\text { nalité }\end{array}$ & $\begin{array}{c}6 \\
\text { Rythme }\end{array}$ & $\begin{array}{c}7 \\
\text { Type } \\
\text { biocéno- } \\
\text { tique }\end{array}$ & $\begin{array}{c}8 \\
\text { Valence } \\
\text { écologique }\end{array}$ & $\begin{array}{c}9 \\
\text { Type } \\
\text { métabo. } \\
\text { lique }\end{array}$ & $\begin{array}{c}10 \\
\text { Réinves- } \\
\text { tissement }\end{array}$ & $\begin{array}{c}11 \\
\text { Type } \\
\text { de } \\
\text { diaspore }\end{array}$ & $\begin{array}{c}12 \\
\text { Elément floristique }\end{array}$ \\
\hline Quercus ilex. & . . & $\mathrm{Pm}$ & $\mathrm{W} 6, \mathrm{~W} 4$ & 3 & $\mathrm{ax}$ & e & Yv & $\mathrm{R}$ & $\mathbf{M}$ & $\mathrm{F}$ & fae & 9 & Méditerranéen \\
\hline Arbutus unedo & - . & Pm & $\mathrm{W} 6, \mathrm{~W} 4$ & 4 & $\mathrm{hx}$ & e & Yv & $\mathrm{R}$ & $\mathbf{M}$ & $F$ & fae & 6 & $\begin{array}{l}\text { Macaronésien- } \\
\text { méditerranéen- } \\
\text { atlantique }\end{array}$ \\
\hline Phillyrea media & . & $\mathrm{Pm}$ & W4 & 3 & $a x$ & e & $\mathrm{Ye}$ & $\mathrm{R}$ & SM & $\mathbf{F}$ & fae & 8 & Méditerranéen \\
\hline Viburnum tinus & · & $P p$ & W4 & 4 & $\mathrm{hx}$ & e & Yv & $\mathrm{R}$ & M & $\mathbf{E}$ & fAE & 6 & $\begin{array}{l}\text { Macaronésien- } \\
\text { méditerranéen- } \\
\text { atlantique }\end{array}$ \\
\hline Smilax aspera & . & Ps & L4 & 4 & $\mathrm{hx}$ & $\mathrm{e}$ & Yv & $\mathrm{R}$ & sM & $\mathrm{D}$ & fae & 6 & Méditerranéen \\
\hline Erica arborea & . & $\mathrm{Pp}$ & W4 & 1 & $\mathrm{nx}$ & $\mathrm{e}$ & $\mathrm{Ye}$ & $\mathbf{R}$ & $\mathrm{sX}$ & $\mathbf{E}$ & Fae & 8 & Afromontagnard \\
\hline Pistacia terebinthus & 3 & $\mathrm{Pp}$ & W4 & 4 & $\mathrm{vz}$ & s & $\mathrm{Ve}$ & $\mathrm{R}$ & $x$ & $\mathbf{E}$ & $\mathrm{FaE}$ & 8 & Méditerranéen \\
\hline Cistus populifolius. & . & $\mathrm{Pp}$ & W4 & 4 & $\mathrm{hz}$ & e & Yv & $\mathbf{R}$ & sM & $\mathbf{E}$ & faE & 8 & Méđiterranéen \\
\hline Rubus ulmifolius & . & $\mathrm{Hs}$ & $\mathrm{H} 4$ & 4 & $\mathrm{vz}$ & d & $\mathrm{Ee}$ & $\mathbf{R}$ & $\mathbf{s X}$ & $\mathrm{D}$ & $\mathrm{FAE}$ & $1-6$ & Européen \\
\hline Teucrium scorodonia & $a$. & ' $\mathrm{Hr}$ & $\mathrm{H} 2$ & 4 & $\mathrm{hz}$ & d & $\mathrm{Ee}$ & $\mathrm{R}$ & $\mathbf{M}$ & $\mathrm{B}^{\prime}$ & FAE & 8 & Atlantique \\
\hline Scilla italica . & . & G & $\mathrm{H} 2$ & 3 & gk & d & $\mathrm{Vv}$ & $\mathbf{R}$ & $\mathrm{sM}$ & C & FAE & 6 & Méditerranéen \\
\hline Coronilla sp. . & . & Pn & W2 & 3 & $\mathrm{vz}$ & d & $\mathrm{Ee}$ & $\mathrm{R}$ & $s \mathrm{X}$ & $\mathrm{B}^{\prime}$ & $\mathrm{FaE}$ & 10 & $?$ \\
\hline Arum italicum & • & $\mathrm{G}$ & H1 & 5 & $\mathrm{hk}$ & d & $\mathrm{Vv}$ & $\mathbf{R}$ & HM & $\mathrm{C}$ & FAE & 6 & $\begin{array}{l}\text { Méditerranéen- } \\
\text { européen }\end{array}$ \\
\hline
\end{tabular}

Polypodium vulgare. $\mathrm{Ch}$

H1

Sedum sp. . . . . .

H1 1 nk

$\mathrm{X}$

B'

faE

Subcosmopolite 
TABEEAU III

Symboles et catégories applicables à l'expression de la structure d'une station de végćtation. En combinant les cinq premiers critères on obtient des types structuraux (voir fig. 2) qui seront ensuite distribués selon leur couverture (sixième critère) (voir fig. 3 ).

1. PORT

Plantes ligneuses, dressée

Plantas lenhosas, erectas

oul rampantes

Plantas lenhosas, trepadeiras ou rastejas

$\mathrm{H} \triangle$ Plantes herbacées, enracinées $\triangle$ Plantas herbáceas, enraizadas

E Epiphytes et croûtes

$\triangle$ Epifitas e crustas

M Bryoïdes

Brióides

2. TYPE DE FEUILLE

- (absente) (ausente)

n aciculaire, écailleuse, subulée acicular, esquamosa, subulada

g $0 \begin{aligned} & \text { graminoïde } \\ & \text { graminóide }\end{aligned}$

a $\diamond$ large, mais petite

larga, mas pequena

h $\bigcirc$ large et grande

larga e grande

$\checkmark W^{\text {composée }}$

q thalloide

talóide

3. TEXTURE DE FEUILLE

O (absente) (ausente)

f pellucide

$\square$ membraneuse

membranos

$x \quad$ sclérophylle

esclerófila

k $\therefore$ succulente ou fongoïde suculenta ou fungóide
4. SAISONNALITÉ VÉGÉtATIVE

$\square$ décidue

IIT semi-décidue

10 semidecídua

e ${ }^{2}$ sempervirente sempre verde

j sempervirente-succulente ou aphylle

sempre verde-suculenta ou afila

5. STRATIFICATION

$6 \quad 10-25 \mathrm{~m}$

$5 \quad 8-10 \mathrm{~m}$

$4 \quad 2-8 \mathrm{~m}$

$3 \quad 0,5-2 \mathrm{~m}$

$2 \quad 0,1-0,5 \mathrm{~m}$

$1 \quad 0,0-0,1 \mathrm{~m}$

c. COUVERTURE

b très faible

escassa

discontinue descontínua

en touffes ou groupes em tufos ou grupos

c continue contínua 
156

nécessaires pour que chaque espèce soit représentée par une formule selon les critères du tableau III, et un répertoire complet des 15 espèces est présenté dans la figure 3 . Ainsi le Quercus ilex répond à la formule W6axe, étant un arbre de moins de 25 mètres de haut, à feuille petite, large et coriace, et sempervirente, cependant que le Smilax aspera correspond à la formule L4hxe, etc. Chacune des 15 espèces a été étalonnée ainsi. Il se trouve souvent que plusieurs espèces ont la même formule en ce qui concerne les cinq premiers symboles (ici le jeune Quercus ilex et le Phillyrea media, par exemple). Il se trouve aussi qu'une même espèce a plus d'une forme structurale (ici le Quercus ilex et l'Arbutus unedo). Il restera à attribuer à chacune des formes structurales l'espace qui lui convient, indépendamment de l'importance relative des espèces ( ${ }^{1}$. Finalement le diagramme de la figure 4 rend compte synthétiquement de l'assemblage final des propriétés structurales de cette station particulière de la chênaie d'yeuse.

Cette mosaïque se prête à des variations qui peuvent sembler infinies. Une autre station de la chênaie d'yeuse présenterait sans doute des variantes (voir DANSEREAU, 1951; fig. 17). Et que dire d'un transect régional où l'échantillonnage irait du marais salé, à la dune, à la garigue, au maquis, à la pinède?

Dynamisme - C'est précisément un tel échantillonnage que nous offre la figure 5 . On y suit les modifications de structure qui accompagnent les changements dans la végétation, qu'ils soient le résultat de la succession progressive qui suit la fin des opérations humaines et qui tend à la reconstitution des équilibres originels, ou de la succession rétrogressive que le labour, le feu, la paissance entraînent.

Ainsi chacun des types de végétation qui apparaît dans la stratégie se caractérise par une composition et une structure particulières.

Le dynamisme est axé sur deux forces concomitantes mais indépendantes. Le remplacement autogène résulte des changements qu'apportent les plantes elles-mêmes à l'équilibre

(1) Ceci ne vas pas sans poser de problèmes. Pcur une discussion détaillée de la méthode, voir Danseread, BUELl \& DAGON (1966).

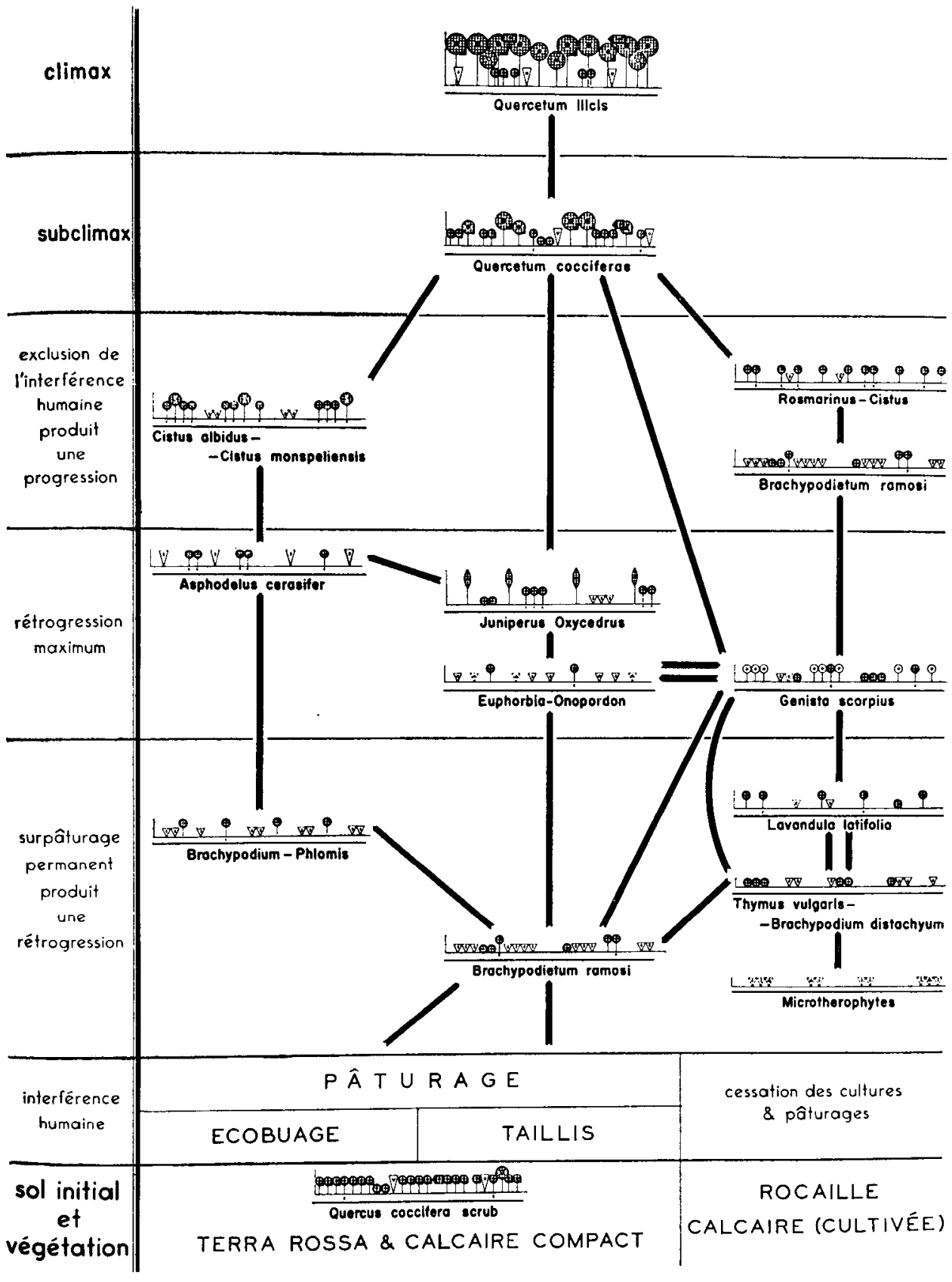

Fig. 5 - Dynamisme de la végétation aux environs de Montpellier, selon BRAUN-BLANQUET (1932, adaptation du tableau 37). Chacune des associations est représentée par un diagramme construit selon la méthode du tableau III, concrétisée par les figures 1-4. 
du milieu; le remplacement allogène résulte de modifications du milieu virtuellement indépendantes de leurs occupants. Ainsi, l'enrichissement $d u$ sol en matière organique, l'augmentation de l'ombre dûe à la croissance des arbres sont des facteurs autogènes, cependant que la sédimentation et l'érosion sont allogènes, comme l'est également le feu et bien entendu l'intervention humaine $\left({ }^{2}\right)$.

\section{LES DÉTERMINANTS DE LA VÉGÉTATION}

Les forces qui déterminent localement et régionalement la mosaïque de la végétation (définie selon les trois paramètres ci-dessus) sont la phylogénie, la chorologie, le climat, le sol et l'histoire. Sans vouloir analyser ces facteurs en détail, il importe à une solide définition et à une classification mondiale des structures de les prendre comme points de repère.

Phylogénie - La sélection naturelle ne saurait se mesurer qu'en termes écologiques. En effet l'ajustement des espèces végétales et animales à un milieu stable ou changeant s'accomplit par des innovations et des remaniements génétiques dont l'aptitude d'une part et l'efficacité d'autre part sont constamment mises à l'épreuve par le milieu. Les plantes qui, à un moment et un endroit donné, sont les mieux douées comme agents de transformation des ressources du milieu deviennent dominantes et canalisent dans leurs tissus une portion importante de l'énergie ambiante. Certains genres se sont montrés extraordinairement productifs et adaptables. Ainsi, les pins, les chênes, les hêtres, les pâturins, les artémises, riches en espèces, larges dans leur distribution géographique exploitent des climats et des sols très différents. Au contraire, les sorbiers, les arbousiers, les luzules, malgré leurs aires géographiques assez grandes, ne constituent guère de biomasses importantes nulle part.

$\mathrm{Si}$, d'autre part, on considère une station de végétation comme un groupement multispécifique où les ressources sont exploitées différentiellement par chacune des populations

(2) Une discussion beaucoup plus détaillée de cette question dans Dansereav (1956) présentes, on relève des équilibres compétition-coopération très différents, et il faut constater un partage où s'affrontent et s'équilibrent les forces de chacune des espèces (DANSEREAU, 1968). Je me suis efforcé naguère (1952) à repérer les éléments de cette stratégie écologique et à tracer les paramètres du succès écologique et par là de faire la projection des occasions d'évolution $\left({ }^{3}\right)$.

Le fait général dont il faut tenir compte, au sujet de la phylogénie, s'exprime par la loi de l'inoptimum (DANSEREAU, $1956,1957,1966 \mathrm{a})$ : «aucune espèce ne rencontre, dans l'habitat qu'elle occupe présentement, des conditions optimales pour la satisfaction de ses exigences à chaque phase de son cycle». Ceci est illustré d'une façon patente par les arbres (comme le pin parasol) qui exigent pleine lumière au moment de la germination mais qui font une excellente croissance à l'ombre, où cependant ne s'établira pas la génération suivante. L'optimum de germination est dans un champ, celui de la croissance, en forêt.

Cette succession d'exigences plus ou moins contradictoires témoigne de pressions de la sélection naturelle dans le passé tantôt dans un sens et tantôt dans l'autre. Ces empreintes historiques confèrent à la plante une certaine vulnérabilité qui rend telle ou telle de ses fonctions critique. Cette faiblesse peut conduire à l'extinction ou tout au moins à la restriction soit de l'habitat, soit de l'aire. Ainsi il existe maints endroits où les cèdres (les véritables Cedrus) et les Sequoia seraient fort à l'aise de nos jours si des adversités antérieures ne les avaient éliminés. Un bon exemple nous est fourni par l'absence du sapin dans la Serra da Estrela, où les genévriers, les genêts et les bruyères forment un étage arbustif dans un climat probablement forestier.

Chorologie - Il faut donc envisager toujours la possibilité que les espèces les mieux douées pour exploiter les ressources d'un écosystème donné ne sont pas présentes. On peut quelquefois démontrer qu'elles l'ont déjà été, telles le sapin au Portugal, l'épicéa aux Pyrénées (Gaussen, 1940). Or, il est

$\left(^{3}\right)$ Cette expression traduit imparfaitement «evolutionary oppor- 
également apparent que les vicissitudes des migrations (surtout celles de grande amplitude) n'ont aucune relation avec la productivité. En effet, il suffira, au cours des avances et retraites des populations d'une espèce quelconque, qu'elle se tienne juste au-delà du seuil de la survivance sans nécessairement atteindre jamais à une efficacité très grande. Là où les réapprovisionnements par migration sont difficiles, comme dans les îles océaniques (Açores, Nouvelle-Zélande), il peut s'ensuivre un appauvrissement cumulatif tel que très peu de plantes soient capables d'une productivité très grande. J'ai comparé, dans ces îles (1965), le taux de croissance des arbres indigènes et exotiques, où il appert que ces derniers sont des exploitants bien plus efficaces. Il arrive même que certains d'entre eux (Pittosporum undulatum aux Açores, Pinus radiata en Nouvelle-Zélande) se naturalisent avec un grand succès et arrivent à déplacer les indigènes d'une façon qui pourrait fort bien être irréversible.

Une société végétale particulière, donc, à un endroit et à un moment donnés (telle la forêt d'yeuse d'Arrábida) se compose d'émergents phylogéniques et chorologiques que l'histoire régionale a laissés comme résidus de ses vicissitudes.

Climat et sol-Le vieux débat sur l'importance relative du climat et du sol est du même ordre que celui de l'hérédité et du milieu. Ces questions ne sont pas dépassées, certes, mais elles sont heureusement dégagées de leurs implications sémantiques ou sociales et philosophiques (qui ne préoccupent plus que les marxistes et les chrétiens les plus anti-révisionnistes, et anti-réductionnistes).

Il ne semble pas douteux que la distribution géographique totale actuelle d'une espèce puisse nous indiquer certaines barrières climatiques qui semblent s'opposer à sa conquête des territoires qui se trouvent au-delà. Ces coïncidences ont été cartographiées dans un bon nombre de cas: le Rubia peregrina en Europe (SALISBURY, 1926), les Stylidium en Australie (GooD, 1925), l'Acer saccharophorum en Amérique du Nord (DANSEREAU, 1957), le Tsuga canadensis en Amérique du Nord (DAHL, 1964), etc.

Un autre aspect de la distribution géographique qui exige une attention plus rigoureuse est le phénomène de la com- pensation, connu depuis longtemps, mais bien explicité par Boyko (1947). Il s'agit de voir si une espèce donnée, lorsque ses populations approchent de la partie la plus froide de son aire géographique, se trouve alors dans les habitats les plus chauds de la région; si dans la partie la plus sèche elle se trouve surtout dans des habitats plus humides, etc. On peut citer d'innombrables exemples de ces compensations. Quelques monographies, par exemple celle du bouleau jaune (DANSEREAU \& PAGEAU, 1966), nous montrent, station par station, la position écologique des populations locales et la prévalence des compensations à la limite de l'aire.

REY (1960) a proposé un système de coordonnées des valeurs météorologiques qui nous permettent une approximation quantitative de la zone géographique optimum d'une espèce végétale et des diverses zones de compensation (au moins potentielle). Il arrive à des conclusions qui n'auraient pas dû nous surprendre, en démontrant que certaines espèces ne trouvent à peu près pas d'espace optimum dans leur aire de distribution actuelle. Ou bien encore, comme c'est peut-être le cas du Quercus rubra en Amérique, elles n'y trouvent pas de conditions édaphiques convenables puisque cette espèce aurait comme optimum climatique une aire en plein océan Atlantique.

A ces considérations il convient d'ajouter l'apparent changement de signe entre les exigences climatiques et édaphiques de certaines plantes, comme le Danthonia spicata, une graminée des sols les plus secs mais exclusivement confinée à des climats d'assez forte précipitation.

Histoire - Ce que nous donne donc une carte détaillée de toutes les stations connues d'une espèce, comme celle du Betula alleghaniensis (DANSEREAU \& PAGEAU, 1966), c'est un périmètre (et des îlots) de distribution qui s'expliquent peutêtre par une certaine combinaison de la variation météorologique présente; c'est aussi une régionalisation des habitats de compensation (surtout les sols très humides dans la zone la plus chaude et surtout la plus sèche). Pour bien comprendre cette distribution, il faut aussi la comparer à d'autres qui lui sont très semblables (voir l'Acer saccharophorum cartographié par DANSEREAU, 1957, et le Tsuga canadensis par DAHL, 1964). 


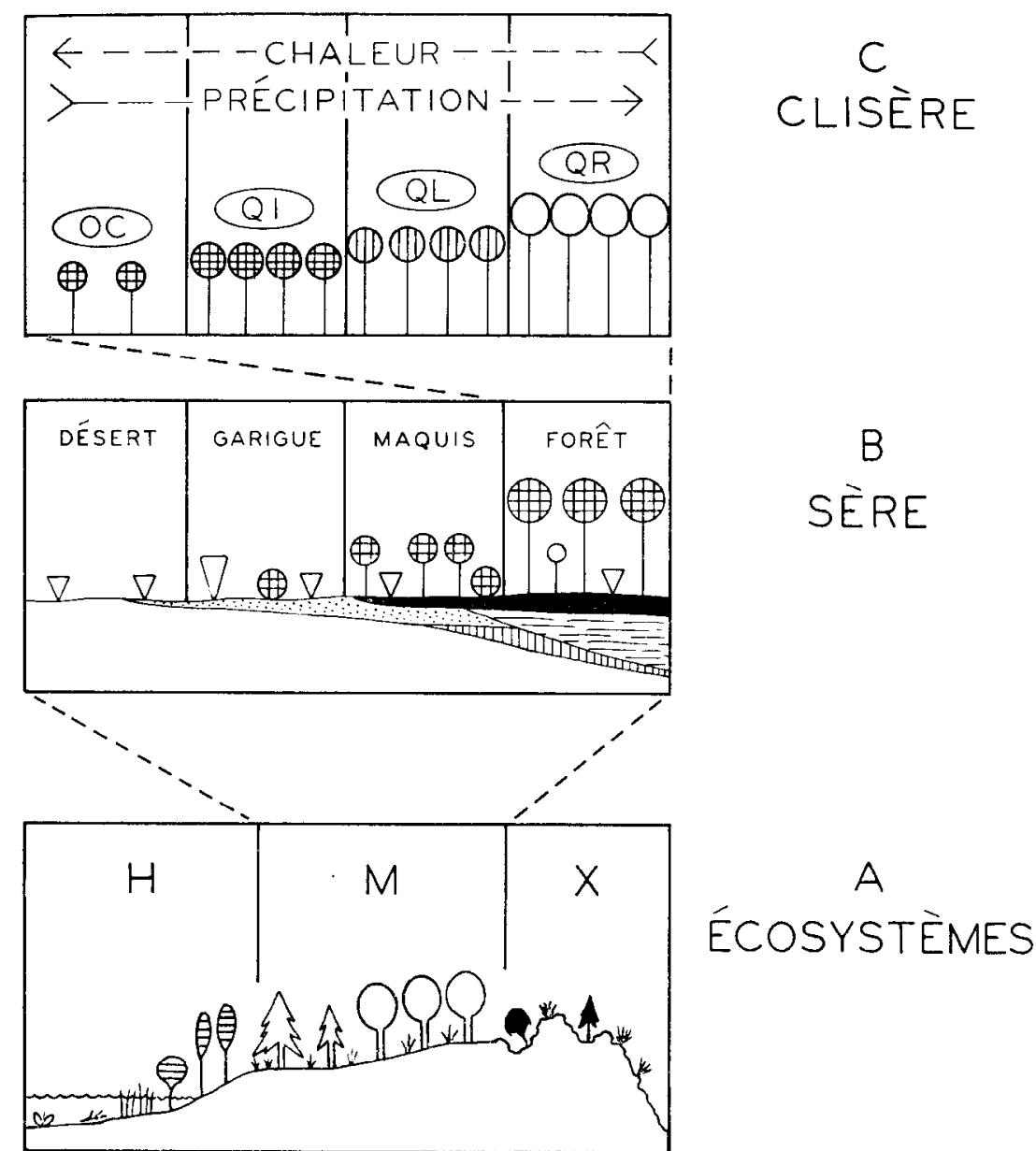

Ces coïncidences d'aires (si admirablement exploitées par Hultén, 1937, pour la flore scandinave), nous donnent une des clefs du passé, si nécessaires à l'interprétation des unités végétales du présent. En ce qui concerne l'Europe atlantique (et même méditerranéenne), et l'Amérique du Nord, les vicissitudes du passé (et surtout du Quaternaire) nous expliquent les équilibres et les aberrations du présent.

Ce n'est pas l'endroit de considérer trop attentivement la question génétique qui pourtant se pose à deux niveaux. Les populations actuelles d'une espèce à grande distribution géographique (même si les taxonomistes ne reconnaissent ni sous-espèces, ni variétés) sont-elles génétiquement homogènes? Non, bien entendu, et il est assez clair que (par introgression ou autrement) les populations périphériques (qu'elles fréquentent ou non des habitats de compensation) sont souvent génétiquement différentes. (Pour l'érable à sucre, voir DANSEREAU \& LAFOND, 1941; DANSEREAU \& DESMARAIS, 1947 ; DESMARAIS, 1952). La seconde question à laquelle toute réponse est spéculative est la suivante: les populations actuelles d'une «espèce» ont-elles les mêmes exigences édapho-climatiques (et par conséquent les mêmes aptitudes physiologiques) que leurs ancêtres? Je m'étais posé cette question au sujet des trois espèces de pins qui survivent actuellement dans la région des Grands-Lacs et du St.-Laurent (DANsereAU, 1953). Je ne trouvais pas moins de sept hypothèses compatibles avec les faits connus de leur distribution et leur abondance présente et post-glaciaire.

La figure 6 tente de résumer ce qui précède au sujet des déterminants de la végétation, tout au moins dans la mesure où cela est nécessaire aux besoins de l'étude structurale.

Le panneau des écosystèmes (A) fait voir une gamme très simplifiée des associations végétales de la région d'Arrábida, telles qu'elles se présentent dans les habitats les plus secs ou xériques (dunes, rochers, falaises) et les plus humides ou hygriques (rivière, marécage, berge) et finalement dans le secteur mésique, constamment bien drainé. Il n'est pas dit que la végétation ou le site lui-même soient nécessairement stables.

Le panneau de la sère (B) montre quelques étapes du développement concomitant de la végétation et du sol sur un
Fig. 6-- Les contrôles de la végétation aux environs d'Arrábida.

A - Les écosystèmes hygrophytiques (H) occupant le segment inondé et humide du paysage; les écosystèmes xérophytiques (X) le segment hyperdraîné (e. g., la dune) ou imperméable (e. g., la falaise); cependant que les terres hautes et bien draînées sont mésophytiques $(\mathrm{M})$.

B --- Le dynamisme dans chaque écosystème permet un remplacement plus ou moins ordonné et régulier de communautés végétales les unes par les autres (formant une sère), à mesure que le sol lui-même se différencie: ains le segment $M$ du panneau A sera cccupé sucessivement par une sorte de désert que recouvrira bientôt la garigue, puis le maquis et enfin la forêt d'yeuse.

$\mathrm{C}$ - La clisère montre également un système de substitution mais au cours de changements climatiques: la forêt d'yeuse (QI) à laquelle aboutit la succession du panneau $B$ a eté remplacée dans le passé et le sera dans l'avenir selon les augmentations-diminutions de chaleur et de précipitation: en période plus chaude par la savane d'olivier-caroubier (OC); en période plus froide et humide par les chênes marcescents (QL) ou éventuellement par les chênes décidus (QR). 
site mésique (le $M$ du panneau $A$ ) : une roche-mère peu altérée n'abritera, au début, que des végétaux isolés (une sorte de désert), mais le site sera bientôt envahi par une garigue basse, à laquelle se substituera un maquis dont les taches d'ombre offriront un microclimat favorable à l'invasion forestière, celle de la chênaie d'yeuse qui ne sera pas déplacée de longtemps, car la sère a atteint son terme. Les horizons du sol, émergeant de la roche sous-jacente, marqueront une différentiation de plus en plus grande jusqu'à ce que le sol zonal corresponde au climax de la végétation.

Le panneau de la clisère $(\mathrm{C})$ fait voir un déplacement de plus grande envergure, car il implique des périodes de temps plus longues au cours desquelles (dans l'avenir comme dans le passé) des augmentations ou diminutions de chaleur et de précipitation conđuiraient au remplacement de la chênaie d'yeuse (QI) en temps plus sec et plus chaud par la savane d'olivier-caroubier (O-C) ou, au contraire, en temps plus froid et humide par la chênaie marcescente ou semi-décidue (QL) et même par la chênaie décidue $(\mathrm{QR})$.

\section{LES DIMENSIONS STRUCTURALES}

Il était indispensable d'esquisser cet arrière-plan de principes et de processus qui gouvernent la végétation avant de mettre au foyer la dimension exclusivement structurale et de proposer une double classification, celle des formations qui constituent des prototypes et celle des classes-de-formation (ou classes-de-végétation). Ces deux classifications répondent à des exigences et des usages fort différents, mais doivent être basées sur les mêmes critères.

Je me suis efforcé de justifier ailleurs (surtout 1951, 1958, 1968) le choix des critères utilisés au tableau III et dans les diverses figures. Avec l'aide de mes collaborateurs BUELL et DAGON (1966), j'ai passé en revue les améliorations de mon système qu'ont proposé divers investigateurs qu'intéresse une plus grande précision et/ou une exigence mathématique plus rigoureuse. J'y ai reconnu l'aide considérable que j'avais reçue de l'United States Army Corps of Engineers et particulièrement de $M$. Warren Grabau, le directeur, à Vicksburg (Mississippi), de l'équipe d'Analyse du Terrain.
Les questions posées dans ce mémoire et celles qui découlent de mes entretiens avec les écologistes, géographes et ingénieurs qui sont à l'affût de meilleures méthodes descriptives ne sont pas en jeu dans le choix et l'application des critères qui vont servir aux deux fins présentes. Ainsi une échelle permettant de représenter l'espacement des tiges nous offrirait un raffinement superflu pour le moment. Il en va de même de certains traits particuliers tels la présence de racines adventives (voir à ce sujet les publications mentionnées plus haut).

Le terme formation était déjà employé par GRISEBACH (1877-78) et par DRUdE (1890), SCHIMPER (1903) et WARMING (1909) et repris par Huguet DEL VILLAR (1929). C'est un terme purement descriptif et physionomique $\left({ }^{4}\right)$ qui s'applique en général à l'aspect de la végétation. Il est bon de lui conserver sa signification compréhensive et à diverses fins: ainsi on parlera d'une formation arborescente, d'une formation herbacée, d'une formation épineuse ou succulente, ou encore d'annuelles ou de lianes; on fera même allusion à une formation de chênes, de pins, d'aulnes, de cistes, de quenouilles, de graminées, etc: Or, même dans ces derniers cas où l'identité taxonomique est révélée, en réalité c'est la dimension physionomique qui est en jeu.

Retenant donc le terme de formation à multiples fins, il importe de distinguer des unités structurales pour des fins plus précises. Je propose donc ici, reprenant mon schéma de 1958 pour mieux le définir, une double classification: celle des protoformations dont l'application est universelle sans être déterminée par des critères géographiques, climatiques, édaphiques ou physiologiques, et celle des classes-de-formation qui, au contraire, correspond à des zones bioclimatiques.

Protoformations - Je me suis efforcé dans les pages précédentes de justifier le besoin d'une classification structurale des végétations qui ne doive rien, d'une part, à la taxonomie et, d'autre part, à l'écologie. Seule une description de la masse végétale basée sur son anatomie et sa physiologie et non pas sur son identité et sur son adaptation répond à cette

(4) Malheureusement fort mal compris et appliqué par CLEMENTS (1936) 
exigence en permettant une juste corrélation des propriétés de la végétation et des éléments déterminants du milieu.

J'ai donc proposé, dès 1958, un tableau universel, basé sur la distribution verticale et horizontale des plantes ligneuses et herbacées. Pour distinguer cette classification de celle des classes-de-formation (que SCHIMPER, 1903, 1935, appelait «Formationstypen»), je les ai dénommées «types-de-formation» (DANSEREAU \& ARros, 1959; DANSEREAU, 1959, 1966b), et en anglais «formation-types». Cette désignation, malheureusement, continue d'engendrer une certaine confusion et c'est pourquoi il me semble plus judicieux de nommer maintenant ces unités «protoformations» (en anglais, «protoformations»; en portugais, «protoformações»; en espagnol, «protoformaciónes»; en italien, «protoformazioni»; en allemand, «Protoformationen»).

Pour la même raison, et en dépit du reproche trop souvent adressé aux biogéographes qui créent des néologismes, j'ai remplacé la nomenclature qui apparaît dans mes ouvrages précédents et qui est donnée dans la colonne des synonymes, au tableau IV. Celui-ci ne diffère pas essentiellement de mon tableau vi de 1958 et de mon «Master-Table C» de 1966b; ni de ceux utilisés dans plusieurs de mes publications au cours de cette période. A l'usage, toutefois, c'est-à-dire en constatant la réaction de mes étudiants et en écoutant les commentaires de mes collègues, je me suis résolu aux changements qui apparaissent ici, et qui portent sur la nomenclature. De la sorte, il ne sera plus question de distinguer la steppe, la toundra, le désert comme protoformations et comme classes-de-formation. Ainsi, il ne sera plus nécessaire de désigner les végétations ligneuses basses des environs de Montpellier et du Cabo de São Vicente sous le nom de toundra (ce qui donnait une sorte de vertige à mes collègues méditerranéens), mais plutôt de microthamnion. Je ne songe pas trop à m'excuser de l'allure pseudo-classique de cette nouvelle nomenclature, un mélange sans vergogne de grec et de latin; mais j'accueillerai avec plaisir les suggestions des linguistes.

La hauteur et la couverture des plantes ligneuses et des plantes herbacées sont seules en jeu. Les limites qui leur sont assignées comportent un certain arbitraire et d'autres chercheurs ont préféré d'autres valeurs (voir KücHLER, 1967). J'ai 
TABLEAU IV

Les dix protoformations. Hauteur et couverture moyenne (en mètres), pas nécessairement maximum. Les «classes» se rapportent à la stratification et à la couverture du tableau III. D'après DANSEREAU, 1958, mais modifié quant à la nomenclature. Les synonymes donnés en français, en anglais et en portugais ont été employés dans des publications précédentes (Dansereau, 1958, 1959, 1966b; Danserea \& ARros, 1959; Dansereau, Buell \& Dagon, 1966).

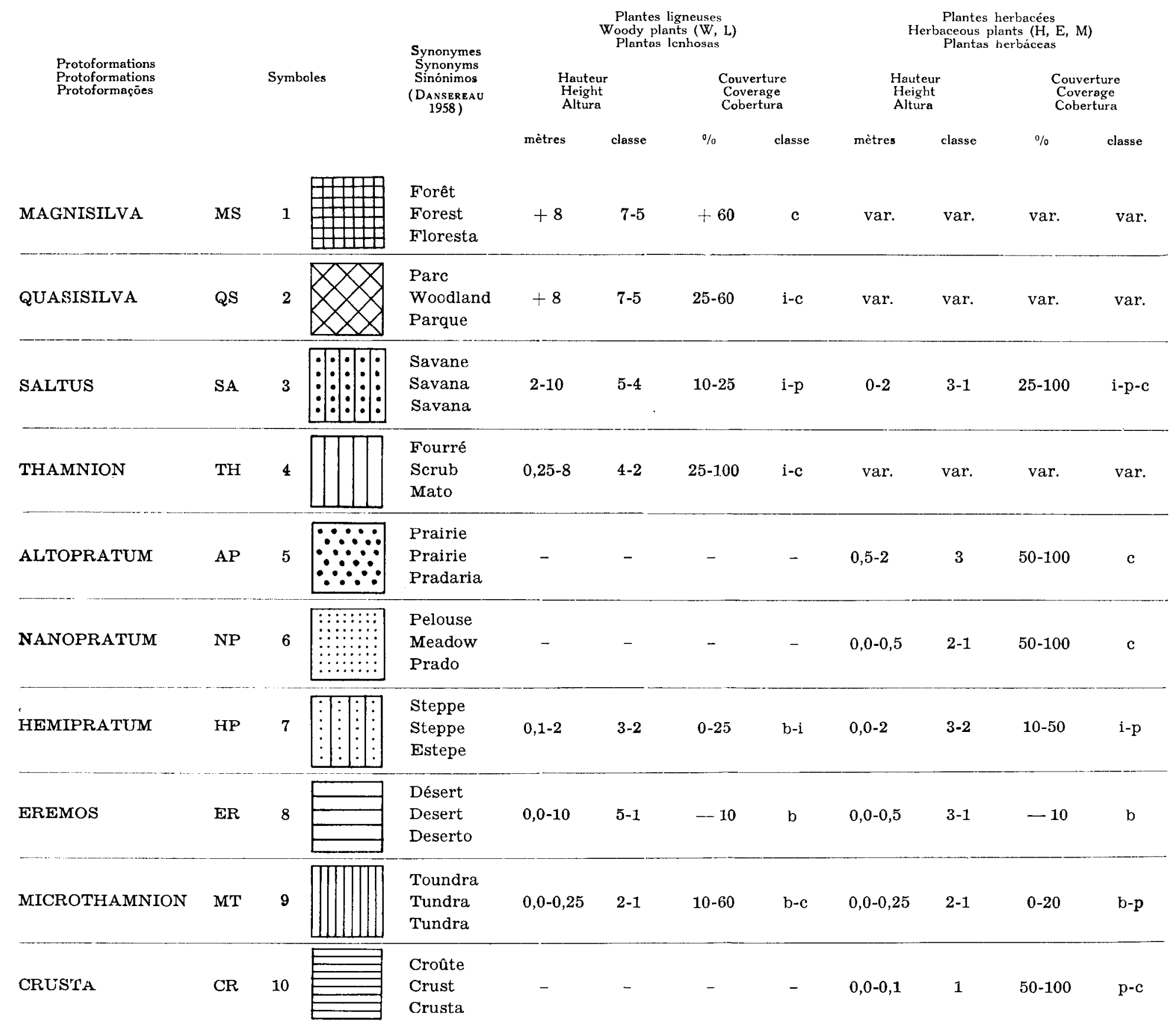


cru utile, pour l'usage de cette classification dans des tableaux, sur des diagrammes, des fiches perforées ou des cartes, de proposer trois séries de symboles et de coordonner avec le tableau IIr.

Je me dois d'ajouter que depuis 1951 je crois avoir été très attentif aux critiques qu'on a faites de mon système de représentation et de classification de la structure de la végétation. Il m'a semblé prudent de laisser passer plusieurs années avant d'effectuer des changements, afin que la preuve de leur urgence soit faite (préférablement par d'autres que moi). Par conséquent je n'ai sérieusement altéré ma proposition de 1951 qu'en 1958 et les nouvelles formules de 1966 (DANSEREAU, BUELL \& DAGON) sont plutôt des expériences qu'autre chose.

Les figures 18 et 19 de mon «A universal system for recording vegetation» (1958) représentent toujours validement les catégories proposées au présent tableau IV. Ces figures avaient été utilisées de nouveau dans un article en français dans «Vegetatio» (DANsEREAU \& ARros, 1959; pls. $\mathbf{D}$ et $\mathrm{E})$.

J'ai assemblé dix photographies qui illustrent par un exemple chacune des protoformations (pls. I à v). On verra immédiatement que certaines d'entre elles ont un caractère transitoire, cependant que d'autres sont permanentes, soit dans leur habitat, soit dans leur région. Ainsi la magnisilva de la planche I, A, est une forêt vierge, marquant le terme de l'évolution de la végétation dans le nord du Québec. Il en va de même (quant à la stabilité) du thamnion canarien, de l'altopratum néo-zélandais et du microthamnion gaspésien. D'autre part, la quasisilva portugaise est remaniée par l'homme, le saltus ouest-africain est récemment incendié et l'hemipratum congolais est surpâturé. Le nanopratum ghanéen, l'eremos brésilien et la crusta brésilienne occupent des sites édaphiques assez particuliers.

Il serait facile de montrer en parallèle à chacun de ces exemples quantité d'autres cas où la protoformation est exactement la même mais où les conditions climatiques et édaphiques sont tout autres. J'en citerai quelques-unes afin de réaffirmer le caractère fondamental de l'unité structurale. 
1. Magnisilva. Forêt de palmiers amazonienne, forêt de chênes rouvres portugaise, forêt d'araucarias brésilienne, forêt de lauriers canarienne, plantation de caoutchouc.

2. Quasisilva. Forêt claire ouest-africaine, parc de pins jaunes du Nevada, parc d'eucalyptus australiens, palmeraie inondée du nord-est brésilien, taïga ouverte sibérienne, parc subalpin pyrénéen, orangeraie.

3. Saltus. Savane guinéenne, savane sahélienne, campo cerrado brésilien, savanes du «muskeg» canadiens, «matagouri» néo-zélandais, pinède californienne à Pinus coulteri, olivette andalouse.

4. Thamnion. Aulnaie américaine, «sagebrush» du Wyoming, «dornveld» sud-africain, «escobonal» canarien, maquis méditerranéen, «chaparral» californien, plantation de thé.

5. Altopratum. «Puszta» hongroise, prairie de l'Iowa, typhaies européennes, américaines, tropicales, phragmitaies du monde entier, «savanes» à Pennisetum de l'Afrique tropicale, champs de canne-à-sucre et de maïs.

6. Nanopratum. Pelouses alpines (Amérique, Europe, Asie), spartinaie intertidale de la côte atlantique, scirpaie des bords de tourbières américaines, pelouses des jardins.

7. Hemipratum. Steppe russe, «short-grass prairie» du Nebraska, certaines garigues méditerranéenes, les laves dures («pahoehoe») récemment colonisées (Hawaii, Congo).

8. Eremos. Beaucoup de rivages rocheux, de dunes nonconsolidées, de carrières récemment abandonnées, de champs vagues, chantiers, etc.

9. Microthamnion. Outre les toundras arctiques et alpines, les formations rases de bruyère en Europe, de thym dans les pays méditerranéens, le «matorral» espagnol à Cistus crispus, certains jardins japonais où dominent, les «bonsai».

10. Crusta. Les revêtements d'algues brunes-fucoïdes dans la ceinture intertidale, les tapis flottants de Lemna sur les étangs, les manteaux de lichens (Stereocaulon) sur les laves d'Hawaii et des Açores, sur les falaises et sur les murs des vieilles maisons de pierre, les algues et les mousses sur les palissades de bois, sur les pavés des rues.

En voilà assez pour évoquer la variété des substrats écologiques susceptibles d'abriter les protoformations.
Il est clair que sur un site initialement nu il est possible que les protoformations se remplacent dans un ordre plus ou moins prévisible. Etant donné la différence de masse de la matière vivante impliquée dans les protoformations cette succession a une importance considérable. J'ai souvent attiré l'attention là-dessus (dès 1946, et bien souvent depuis: 1956, 1957, 1958, 1961). Ce dont il importe de se garder, c'est d'une ordonnance nécessairement linéaire, allant du $n^{0} 10$ au $n^{\circ}$ 1. En effet diverses successions suivront des ordres divers, ainsi 10-6-4-1, ou 8-9-6-3-2-1, ou 7-4-2-1, etc.

Les classes-de-formation - Je ne tenterai pas de décrire ici pleinement les 20 classes-de-formation qui se partagent les surfaces terrestres du globe. Dans une tentative de renouveler les classifications antérieures, particulièrement celles de SCHIMPER \& VON FABER (1935) et de BROCKMANNJERosch (1954), je m'en étais tenu le plus étroitement possible à des unités structurales, et avais reconnu 16 unités seulement $(1957,1958)$. Les critères que je m'étais fixés à ce moment n'ont guère changé.

Or, les dix protoformations, appliquées empiriquement aux paysages du monde, ne sont clairement plus suffisantes. Il faut subdiviser les magnisilva plusieurs fois; il faut reconnaître divers thamnion et divers saltus si l'on espère rendre compte de l'accord entre la végétation dominante et l'équilibre climat-sol. Car je continue de suivre ScHIMPER en reconnaissant que chacune des zones caractérisées par l'une des classes-deformation est déterminée d'abord par un régime climatique et pédogénique d'un type et d'une intensité particuliers.

Alors comme maintenant il m'a aussi semblé important de caractériser chacune des classes-de-formation par certaines formations végétales «secondaires» (tels les tourbières dans le cas de la forêt sempervirente aciculifoliée, les maquis dans le cas de la forêt sclérophylle, etc.).

Les 20 classes-de-formation énumérées au tableau V doivent être décrites en appliquant les critères suivants: description structurale, régime climatique déterminant, régime pédogénique associé, principaux types de végétation des habitats plus secs et plus humides. On cherchera alors à les répartir sur la carte, comme l'a fait BROCKMANN-JEROSCH 
(1954). Une telle description dépasse le cadre du présent mémoire. Elle a été présentée ailleurs et sera élaborée à fond dans un livre sur la végétation du monde.

On peut les repérer dans un triangle climatique (comme je l'ai fait à plusieurs reprises depuis 1949, et notamment en $1953,1957,1958,1964,1965$ ). La figure 7 , en situant les classes-de-formation les unes par rapport aux autres, indique les changements de structure qu'entraînera un réchauffement ou un refroidissement de climat ou encore un assèchement ou une humidification. Sur un tel arrière-plan il est possible

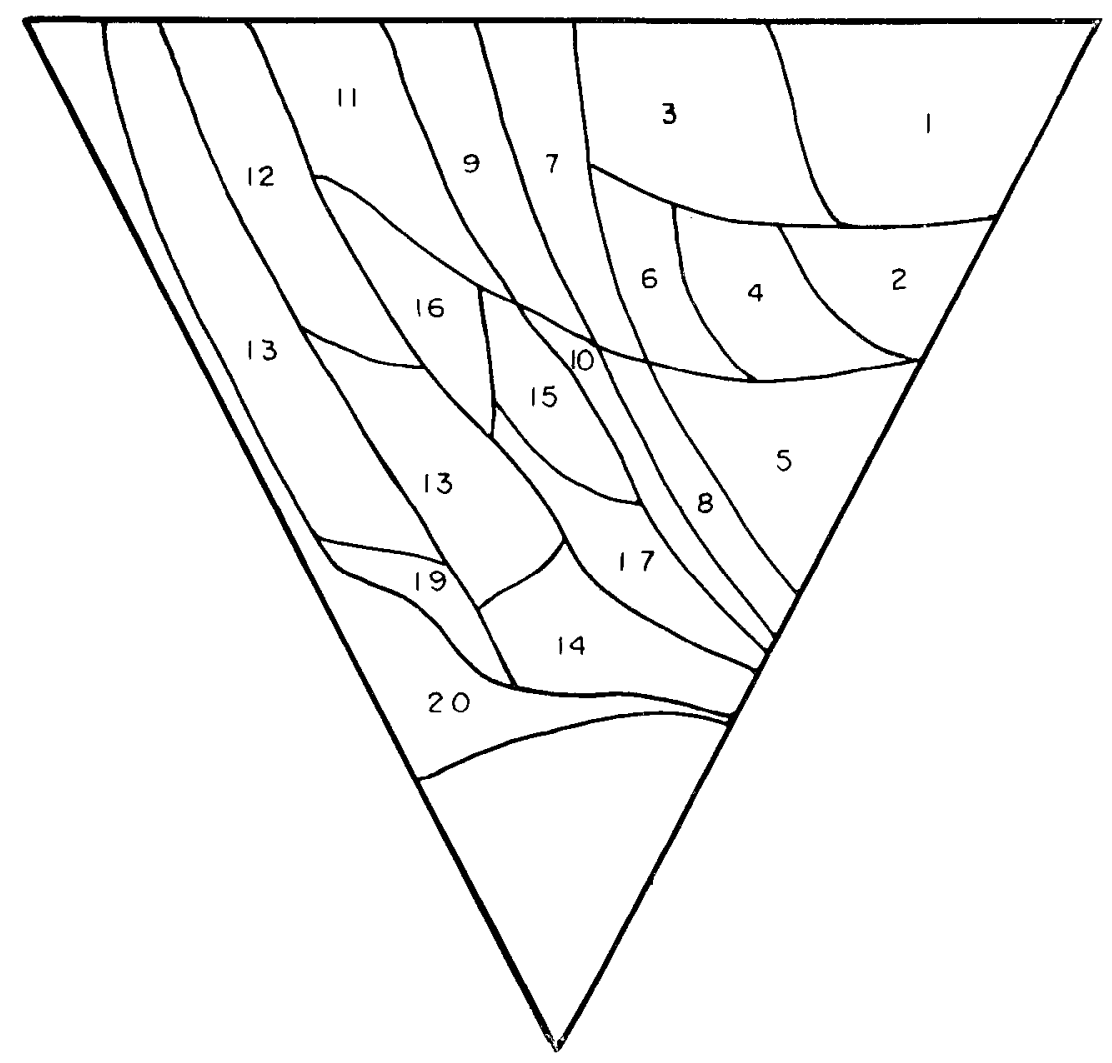

Fig. 7-Triangle bioclimatique montrant la position relative des classes-de-formation (numérotées de 1 à 20 comme au tableau v). Le maximum de chaleur occupe le sommet de la figure, les précipitations croîssent de gauche à droite

$$
\text { (d'après DANSEREAU, 1964). }
$$




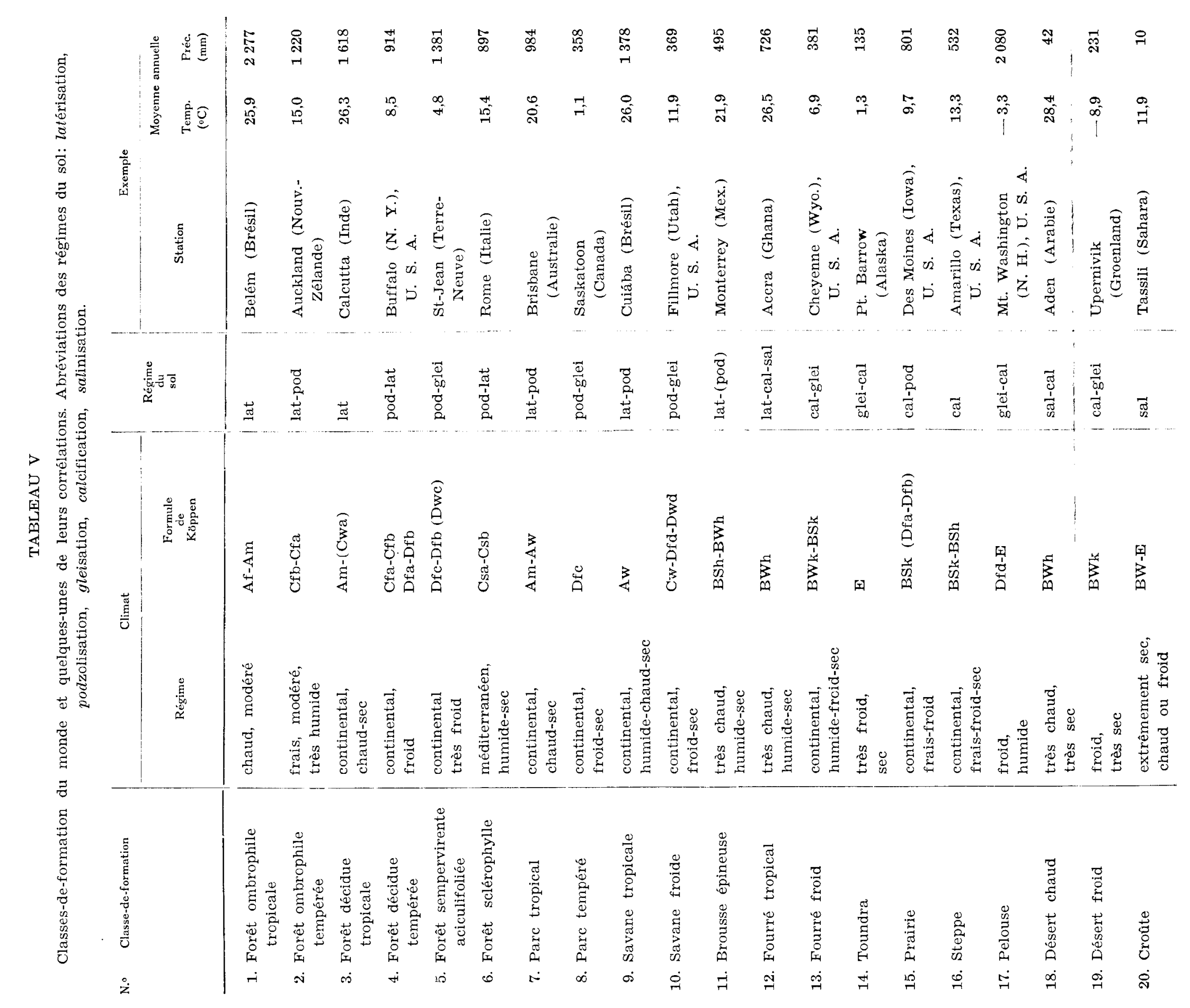


d'indiquer l'adaptation bioclimatique de certaines plantes (voir DANSEREAU, 1953, pour les Magnolia, Sequoia, Artemisia, etc.) ou encore le glissement présent ou passé de diverses elisères comme celle de la figure 6 ou celles de la NouvelleZélande, des Îles Canaries et de la Vallée du St.-Laurent (DANSEREAU, 1964, 1965).

\section{CONCLUSION}

Toute étude de la végétation doit distinguer nettement les propriétés des populations végétales elles-mêmes des conditions écologiques, édaphiques et climatiques avec lesquelles on cherche une corrélation, qu'elle soit causale ou accidentelle. Les phytogéographes (et souvent les géographes eux-mêmes) ont souvent concentré toute leur attention sur la composition de la végétation, sans toujours se rendre compte qu'une énumération d'espèces n'avait guère de valeur descriptive ni explicative. Autrement dit, la connaissance de la flore ne révèle pas plus le caractère de la végétation que l'analyse chimique ne décrit le sol. Un examen indépendant et exclusif des structures de végétation fait voir que les espèces d'une même flore peuvent s'assembler en des structures différentes (une forêt, une savane, un fourré) et que, d'autre part, des espèces de flores différentes peuvent s'assembler en une structure identique (forêt sclérophylle portugaise et chilienne; maquis méditerranéen, chaparral californien et manuka néozélandais).

Le tableau IV propose un répertoire exclusif de 10 protoformations basées sur la distribution verticale et horizontale des plantes ligneuses et herbacées, quelle que soit leur composition, quel que soit le climat et le sol occupés, puisque ce sera précisément cette corrélation qu'on cherchera à établir. Cette classification est applicable à toute station de végétation, qu'elle soit vierge, spontanée, secondaire, cultivée ou artificielle et de quelque étenduc qu'elle soit.

D'autre part, les classes-de-formation sont des unités géographiques, coïncidant avec un climat régional (et souvent avec un sol zonal) et prévalent sur les terres bien drainées du territoire. Il est évident que la classe-de-formation (par exemple la forêt sclérophylle au centre et au sud du Portugal) 
n'occupe pas tout le territoire (voir fig. 6): elle en donne seulement la clef en exprimant l'accord d'un certain développement optimum de la végétation avec le climat ambiant. PIERRE DANSEREAU

\section{BIBLIOGRAPHIE}

BoYko, HUGo (1947) - «On the role of plants as quantitative climate indicators and the geoecological law of distribution». Jour. Ecol. 35(1-2): 138-157.

BRAUN-BlanqUeT, Josias (1928)-Pflanzensoziologie; Grundzüge der Vegetationskunde. J. Springer, Berlin, $\mathrm{x}+330 \mathrm{pp}$.

BraUn-Blanquet, Josias (1932) - Plant sociology (transl. by G. D. Fuller \& H. S. Conard). McGraw-Hill Book Co., Inc., New York, XVIII $+439 \mathrm{pp}$.

Braun-Blanquet, Josias (1964)-Pflanzensoziologie. Grundzüge der Vegetationskunde ( $3^{\text {e }}$ édition). Springer-Verlag, Wien, New York, XIV + $865 \mathrm{pp}$.

BRoCKMANN-JEROSCH, H. (1954) - Plant communities of the world. Map, at 1:20000000, VEB Geographisch-Kartographische Anstalt Gotha.

ClFments, Frederic E. (1936) - «Nature and structure of the climax». Jour. Ecol., 24(1): 253-284.

DAHL, EILIF (1963) - «Present-day distribution of plants and past climate». In: The reconstruction of past environments, ed. by James J. Hester \& James Schoenwetter, Fort Burgwin Res. Center, $\mathrm{n}^{\circ} 3$, pp. 53-60

DANSEREAU, PIERRE (1946) -- «L'érablière laurentienne. II - Les successions et leurs indicateurs». Can. Jour. Res., C 24(6): 235-291; aussi Contrib. Inst. Bot. Univ., Montréal, 60: 235-291.

DANSEReaU, Pierre (1949) - «Introdução à biogeografia». Rev. Brasil. Geogr., 11(1): 3-92.

DANSEREAU PIERRE (1951) - «Description and recording of vegetation upon a structural basis». Ecology, 32(2): 172-229; aussi Bull. Serv. Biogéogr., 8: 172-229 (1953).

DANSEREAU, PIERRE (1952) — «The varieties of evolutionary opportunity». Rev. Can. Biol., 11(4): 305-388.

DANSEREAU, PIERRE (1953) - «The postglacial pine period». Trans. Roy. Soc. Can., 47 (sér. III, sect. V): 23-38.

DANSEREAU, Pierre (1956) - «Le régime climatique régional de la végétation et les contrôles édaphiques». Rev. Can. Biol., 15(1): 1-71.

DANSEREAU, PIERRE (1957)-Biogeography: an ecological perspective. Ronald Press Co., New York, XIII +394 pp.

DANSEREAU, PIERRE (1958) - «A universal system for recording vegetation». Contrib. Inst. Bot. Univ., Montréal, 72: 1-58.
DANSEREAU, PIERRE (1959) — «Phytogeographia laurentiana. II - The principal plant associations of the Saint Lawrence Valley». Contrib. Inst. Bot. Univ., Montréal, 75: 1-147.

DASNEREAU, PIERRE (1961) - «The origin and growth of plant communities». In: Growth in Living Systems, Proc. Symp. on Growth, Purdue Univ. (Indiana), June 1960; ed. by M. X. Zarrow; Basic Books, New York, pp. 567-603.

DANSEREAU, PIERRE (1964) - «Six problems in New Zealand vegetation». Bull. Torrey Bot. Club, 91(2): 114-140.

DANSEREAU, PIERRF (1965) - - «Le contrôle de la végétation dans les îles océaniques». Comunicación al Coloquio «Aportación de las Investigaciones Ecológicas y Agrícolas a la lucha del mundo contra el hambre» (Cons. Sup. Investig. Cient., Madrid), ses. 5, 41 pp.

DANSEREAU, PIERRE (1966a)- - Ecological impact and human ecology». In: Future Environments of North America, ed. by F. F. Darling \& J. P. Milton, Nat. Hist. Press, Garden City, New York, $\mathrm{xv}+767$ pp. (pp. 425-462).

DANSEREAU, PIERRE (1966b) - «Studies on the vegetation of Puerto Rico. I - Description and integration of the plant communities. BUELL, Peter F. and DANSEREAU PIERRE; II - Analysis and mapping of the Roosevelt Roads Area». Inst. Carib. Sci., Spec. Publ., n 1, 287 pp. + maps.

DANSEREAU, PIERRE (1968) - «The functional analysis of plant-communities, a multidimensional grading system». Sarracenia $\mathrm{n}^{\circ} 12$ (sous presse).

DANSEREAU, PIERRE et ARROS, JENö (1959) - «Essais d'application de la dimension structurale en phytosociologie. I - Quelques exemples européens». Vegetatio, 9(1-2): 48-99; aussi Bull Serv. Biogéogr., 22: 48-99.

Dansereat Pierre; Buell, Peter F, and Dagon, Ronald (1966)- «A universal system for recording vegetation. II $-\mathrm{A}$ methodological critique and an experiment». Sarracenia $\mathrm{n}$ " 10, $64 \mathrm{pp}$

DANSEREAU, PIERRE and Desmarais, Yves (1947) - «Introgression in sugar maples-II. Amer. Midl. Nat., 37(1): 146-161.

Dansfreau, Pierre et LAFOND, ANdré (1941) - «Introgression des caractères de l'Acer saccharophorum K. Koch et de l'Acer nigrum Michx». Contrib. Inst. Bot. Univ., Montréal, 37: 15-31.

Dansereau, PIERRE and Lems KoRnelius (1957)-- «The grading of dispersal types in plant communities and their ecological significance». Contrib. Inst. Bot. Univ., Montréal, 71: 1-52.

Dansfreau, Pierre et PAGEAU, GÉRARd (1966) - «Phytogeographia laurentiana. IV - Distribution géographique et écologique du Betula alleghaniensis». Mém. Jard. Bot., Montréal, 58: 1-56.

DESMARAIS, Yves (1952) - «Dynamics of leaf variation in sugar maples». Brittonia, 7(5): $347-387$.

DRUDF, O. (1890) - Handbuch der Pflanzengeographie. J. Engelhorn Stuttgart, $582 \mathrm{pp}$. 
GaUSSEN, HEnRi (1940) - «Le milieu physique et la forêt au Portugal». Trav Lab. Forest., Toulouse, tome IV, vol, I, art. II, pp. 1-49. GOod, RoNALD (1925) - «On the geographical distribution of the Stylidiaceaex. New Phytol., 24: 225-240

GRISEBACH, A. (1877-1878) - La végétation du globe d'après sa disposition suivant les climats. Esquisse d'une géographie comparée des plantes». ('Traduit par P. de Tchihatchef), Librairie J.-B. Baillière et Fils, Paris, tome I: XVI +765 pp.; tome II: VI +905 pp.

HULtÉN, ERIC (1937) - «Outline of the history of Arctic and Boreal biota during the Quaternary Period». Bokförags Aktiebolaget Thule, Stockholm, $168 \mathrm{pp} .+42 \mathrm{pl}$.

KÜCHLER, A. W. (1967) - Vegetation mapping. Ronald Press Co., New York, vi $+472 \mathrm{pp}$.

RAUNKIAER, C. (1934) - The life forms of plants and statistical plant geography. Clarendon Press, Oxford, XVI $+632 \mathrm{pp}$

Rey, PAUl (1960)-Essai de phytocinétique biogéographique. Thèse, Fac. Sci., Univ. Toulouse (Impr. Louis-Jean, Gap), 399 pp.

SAlisbury, E. J. (1926) - «The geographical distribution of plants in relation to climatic factors». Geogr. Jour., 67: 312-335.

SCHIMPER, A. F. W. (1903) -- Plant geography upon a physiological basis. (Transl. by Wm. R. Fisher). Clarendon Press, Oxford, $839 \mathrm{pp}$

SCHIMPER, A. F. W. und FABER, F. C. (1935) - Pflanzengeographie auf physiologischer Grundlage. Fischer, Jena, 1: $\mathrm{xx}+588$ pp.; 2: $\mathrm{xx}+589-1613 \mathrm{pp}$.

VILLAR, E. HUGUET DEL (1929)-Geobotánica. Editorial Labor, Barcelona-Buenos Aires, $339 \mathrm{pp}$

Warming, E. (1909) - Oecology of plants. An introduction to the study of plant communities. Oxford Univ. Press, London, XI +422 pp.

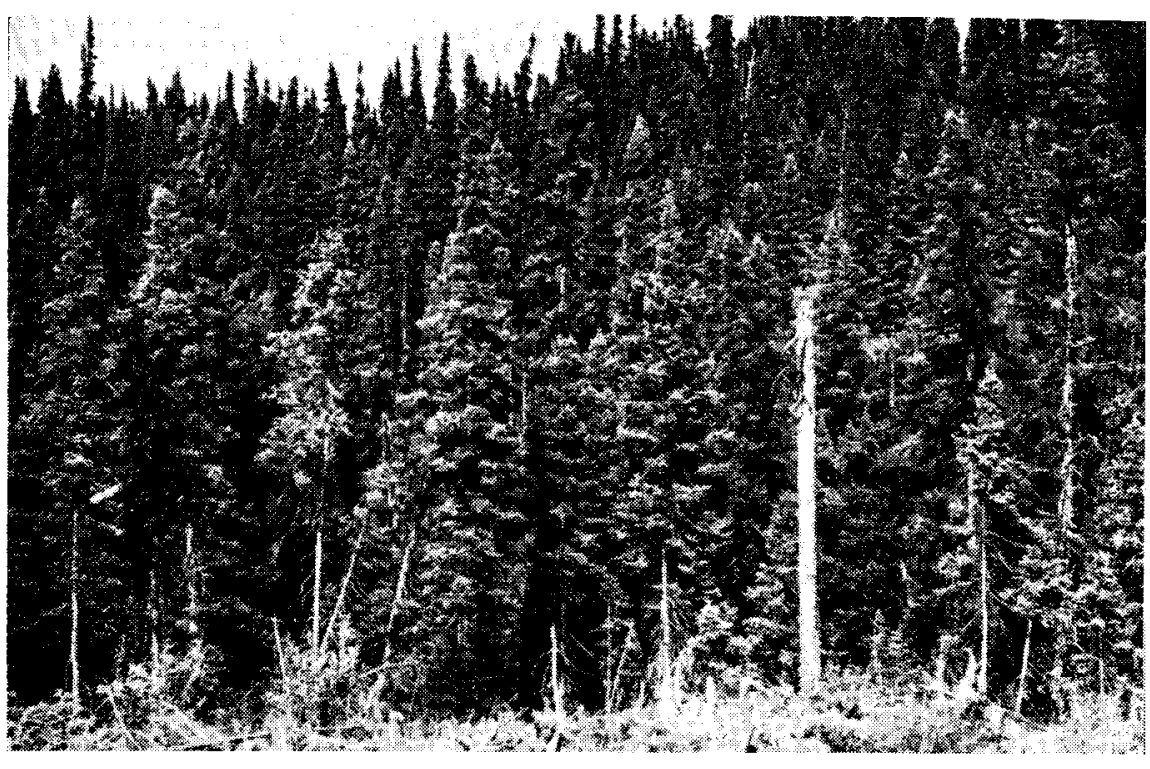

PL. I, A - Magnisilva. Une forêt d'épicéa (Picea glauca) dans la Province de Québec (Canada).

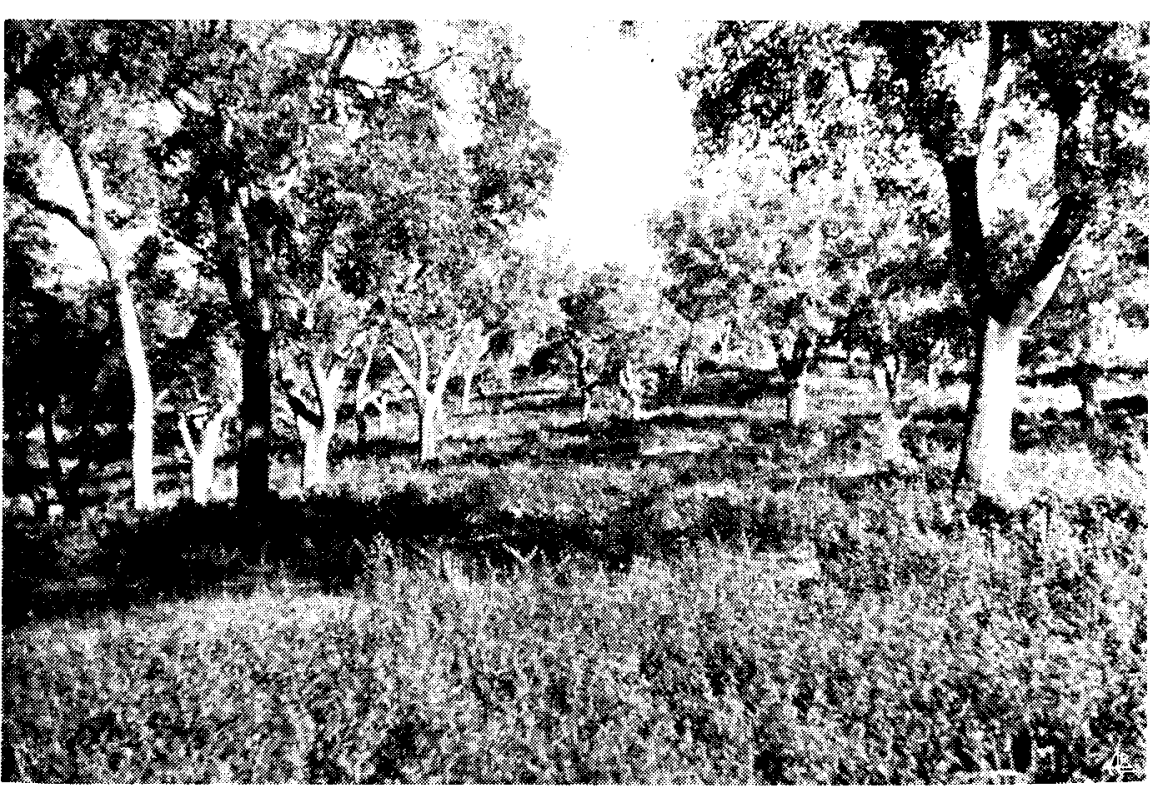

PL. I, B-Quasisilva. Un parc de chêne-liège (Quercus suber) au Portugal. 


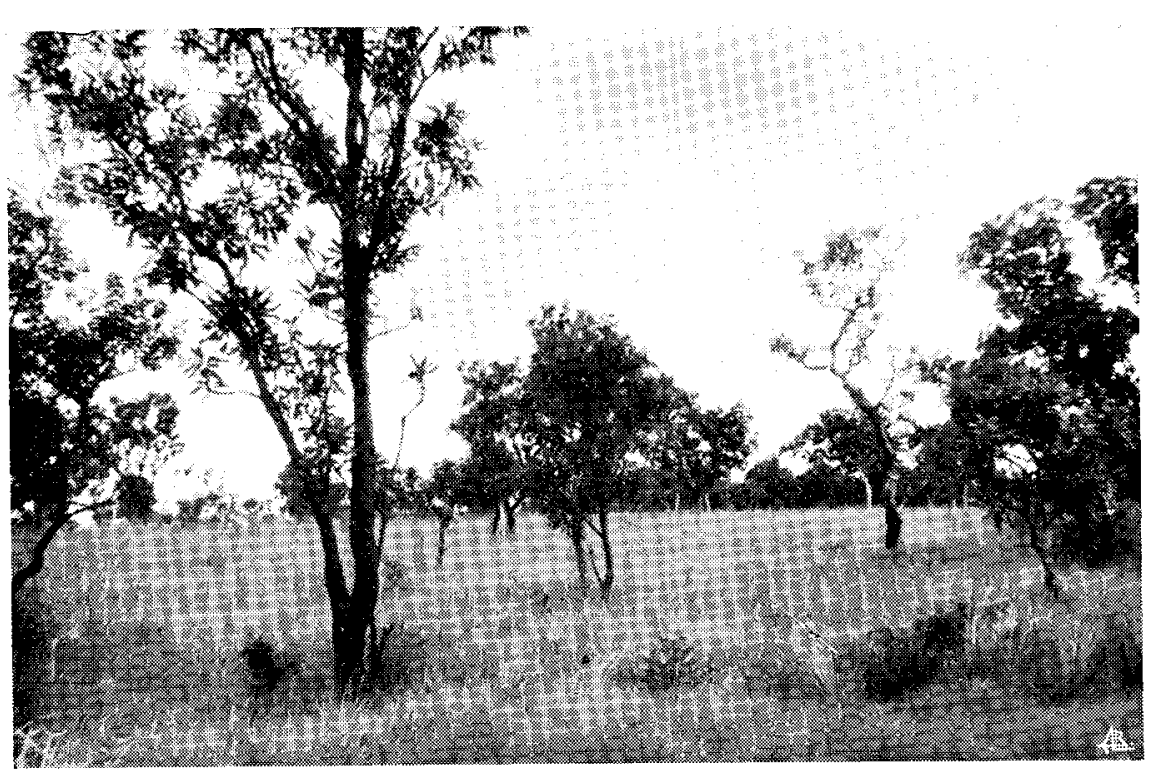

PL. II, A - Saltus. Une savane de lophira (Lophira alata) en Côte d'Ivoire.

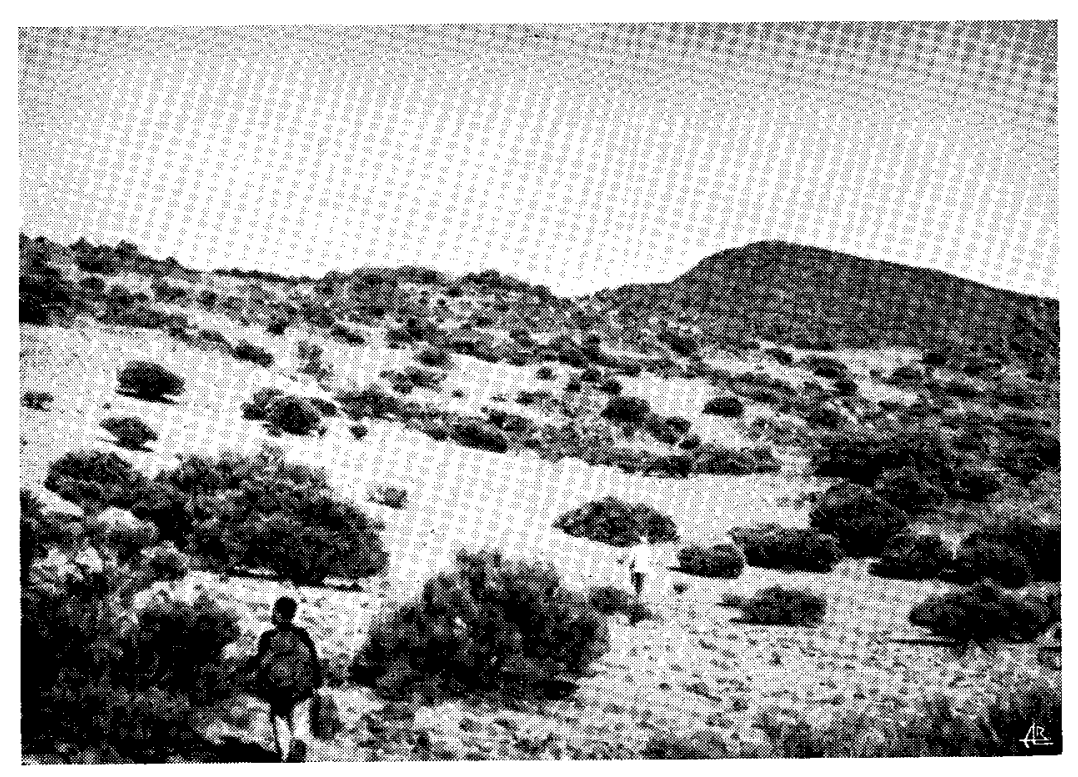

PL. II, B - Thamnion. Un fourré de genêts (Spartocytisus nubigenus) sur le Teyde, aux Iles Canaries. 


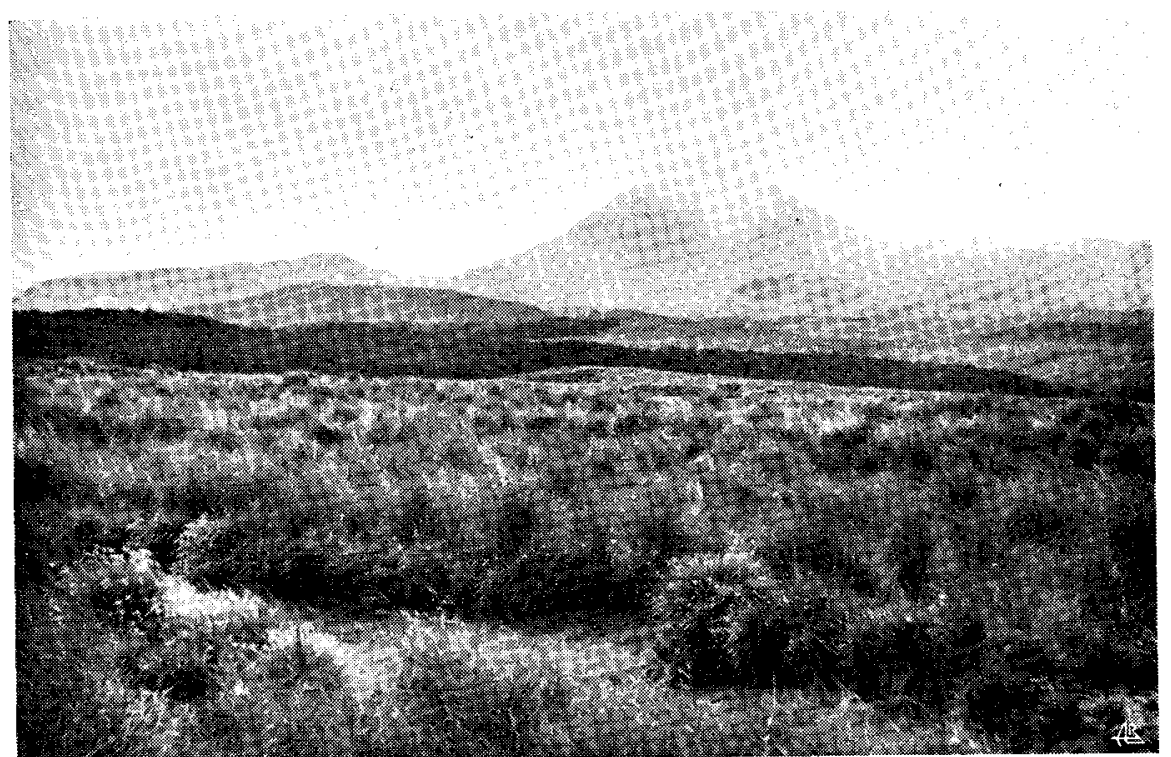

PL. III, A-Altopratum. Un tussockland à danthonies (Chionochloa) en Nouvelle-Zèlande.

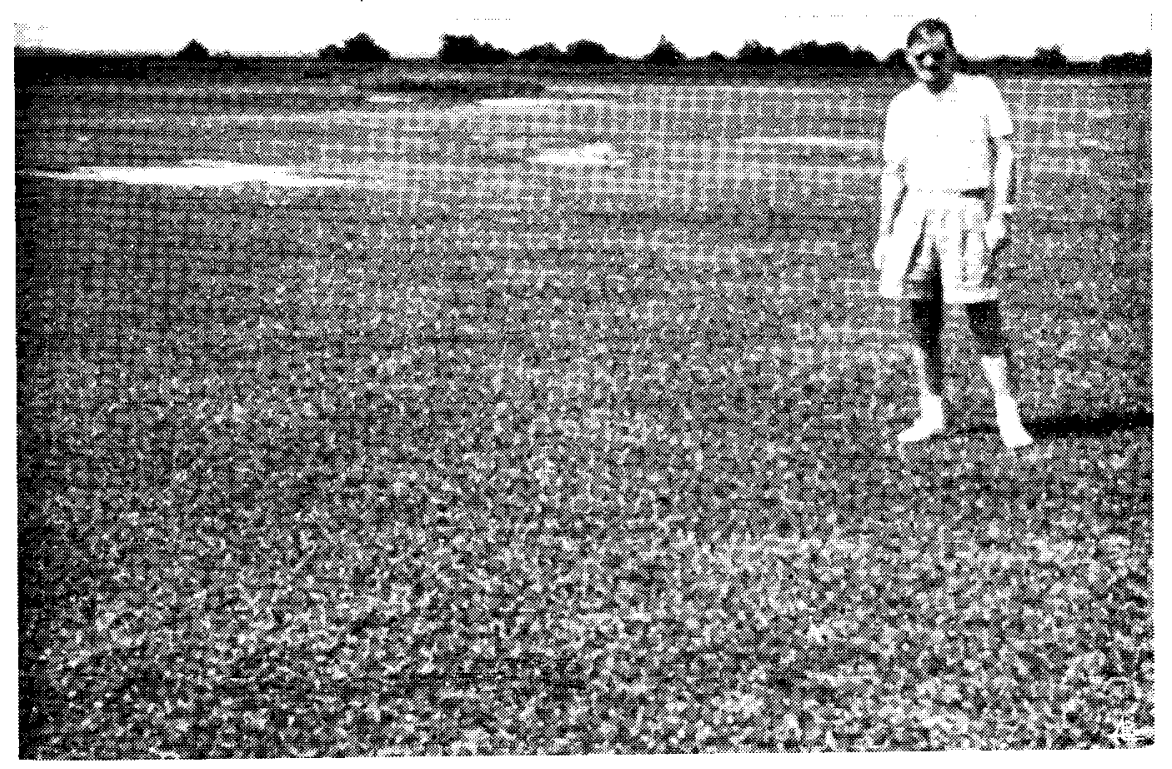

PL. III, B-Nanopratum. Une pelouse rase et succulente (de Sesuvium portulacastrum) sur le littoral de Ghana. 


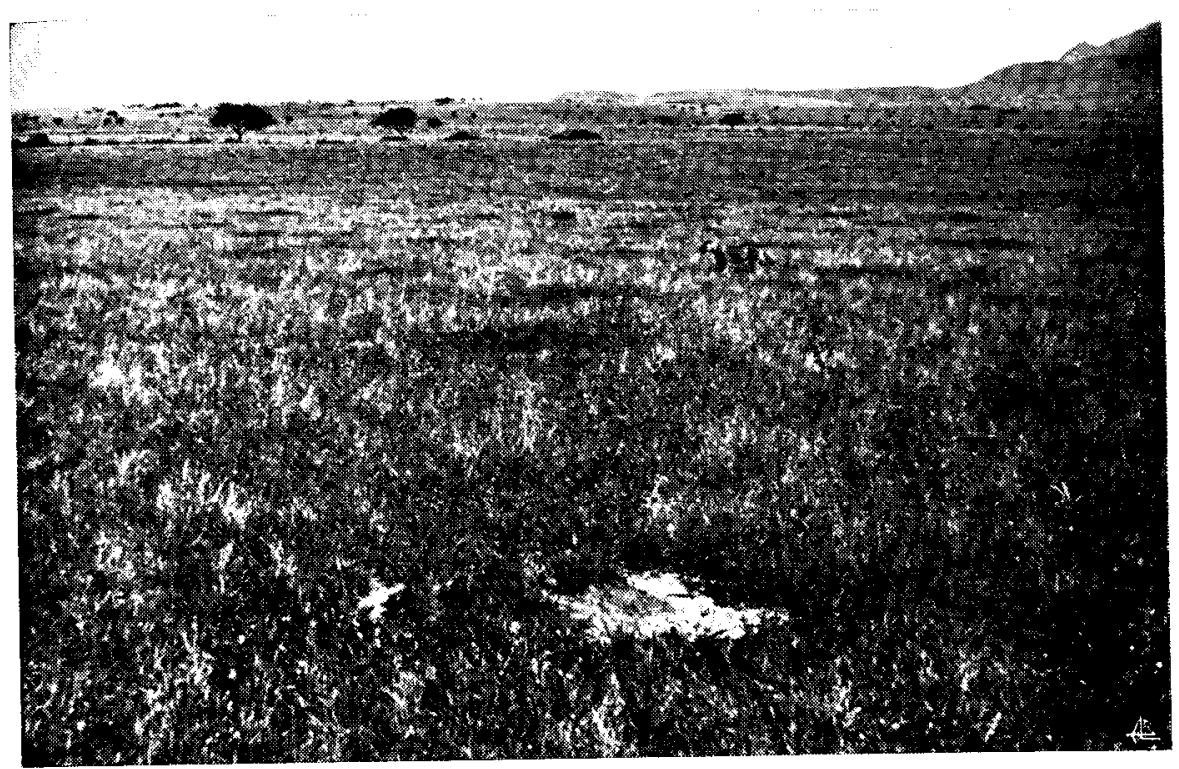

PL. IV, A-Hemipratum. Une steppe à graminées et Carissa dans le Parc Albert, au Congo.

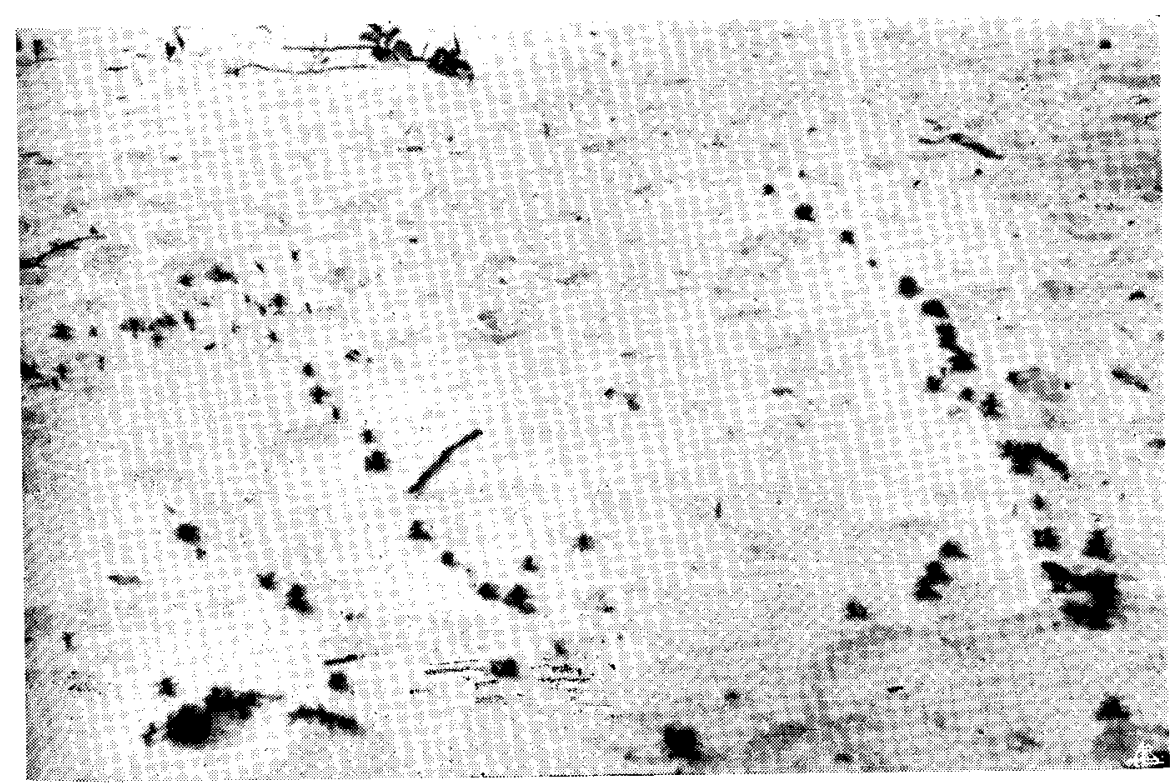

PL. IV, B-Eremos. Un désert littoral avec touffes de Remirea maritima et de Scaevola plumieri, près de Rio de Janeiro, Brésil. 


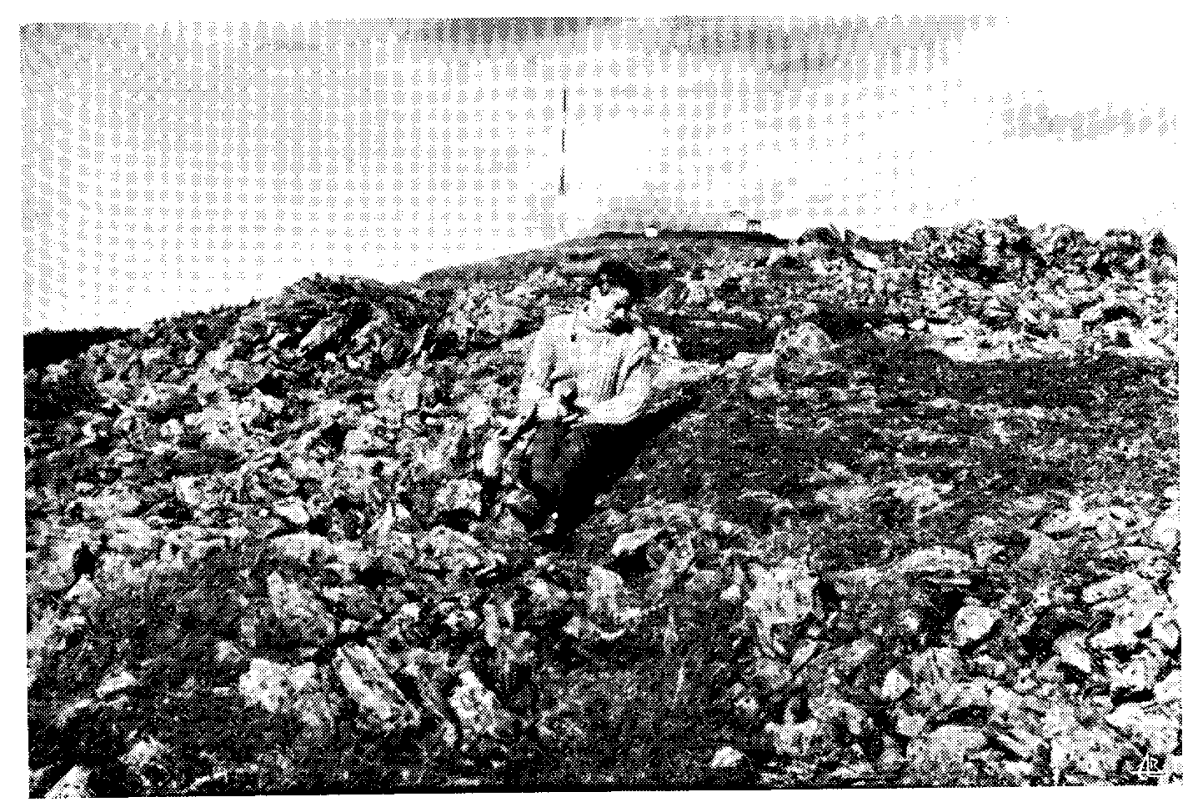

PL. V, A-Microthamnion. Une toundra alpine (à Vaccinium et Diapensia) sur le Mont Logan, Province de Québec, Canada.

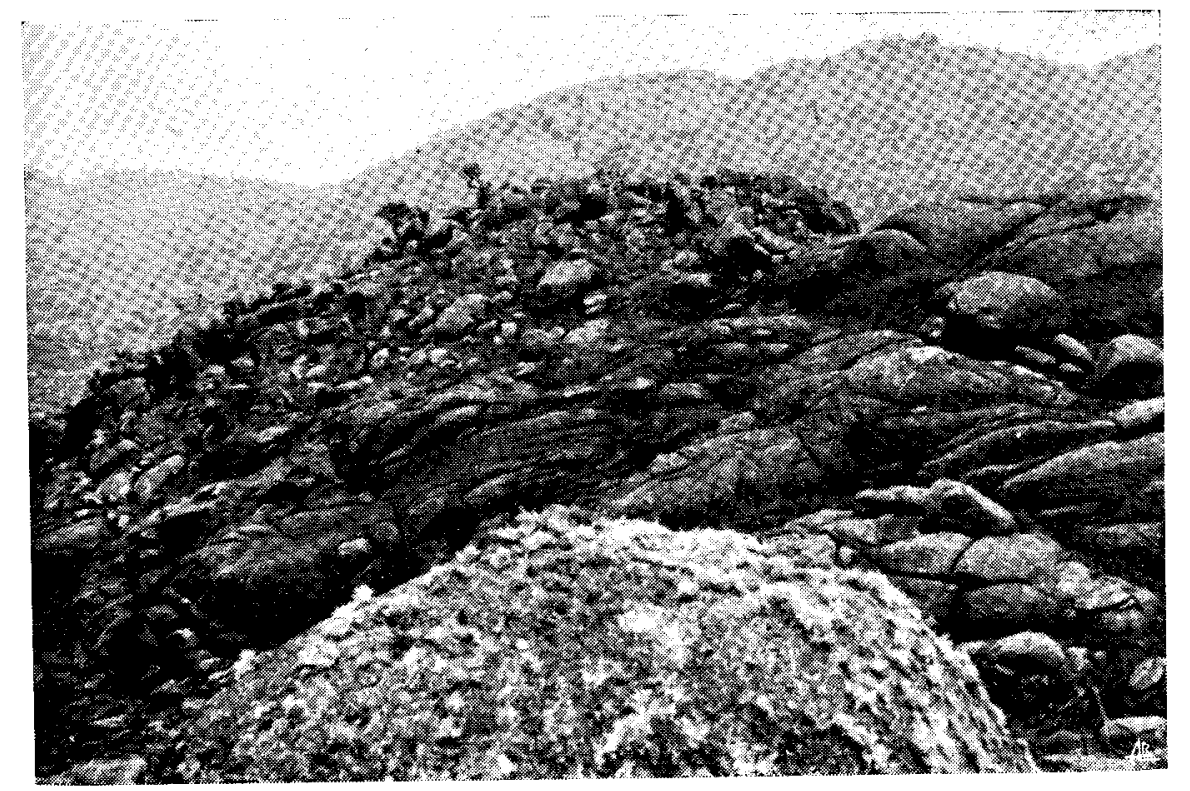

PL. V, B-Crusta. Une croute de lichens, à haute altitude sur le Mont Itatiaia, au Brésil. 